\title{
TAMENESS OF THE PSEUDOVARIETY LSl
}

\author{
J. C. COSTA and M. L. TEIXEIRA ${ }^{1}$ \\ Centro de Matemática da Universidade do Minho \\ Campus de Gualtar, 4700-320 Braga, Portugal \\ Email: jcosta@math.uminho.pt,mlurdes@math.uminho.pt
}

October 3, 2003

\begin{abstract}
AMS Mathematics Subject Classification: 20M05, 20M07, 20M35
The notion of $\kappa$-tameness of a pseudovariety was introduced by Almeida and Steinberg and is a strong property which implies decidability of pseudovarieties. In this paper we prove that the pseudovariety $\mathbf{L S l}$, of local semilattices, is $\kappa$-tame.
\end{abstract}

Keywords: Semigroup; pseudovariety; implicit operation; tameness; local semillatice; infinite word; graph labelling.

\section{Introduction}

A class of finite semigroups $\mathbf{V}$ is said to be decidable if the membership problem for $\mathbf{V}$ has a solution, that is, if there is an algorithm to test whether a given semigroup lies in $\mathbf{V}$. In the applications of the theory of finite semigroups to the theory of formal languages, via Eilenberg's correspondence between pseudovarieties of semigroups and varieties of formal languages [14], many problems in computer science can be formulated in terms of the decidability problem for pseudovarieties of semigroups. For example, the work of Krohn and Rhodes in automata theory in the mid 1960's, leads to the statement of the Krohn - Rhodes complexity problem [15], which may be formulated in terms of the decidability of the iterated semidirect products of the form $\mathbf{A} *(\mathbf{G} * \mathbf{A})^{n}, n \in \mathbb{N}$, where $\mathbf{G}$ is the pseudovariety of all finite groups and $\mathbf{A}$ is the pseudovariety of all finite aperiodic semigroups (i.e., semigroups whose subgroups are trivial).

Decidability of pseudovarieties is not preserved by some of the most common pseudovariety operators, such as semidirect product, Mal'cev product and join [1, 19]. In particular Rhodes [19] has exhibited a decidable pseudovariety $\mathbf{V}$ such that the semidirect product $\mathbf{S l} * \mathbf{V}$ is not decidable, where $\mathbf{S l}$ is the pseudovariety of semilattices. This fact suggested a need to establish and to study stronger conditions, expecting them to be more robust in proving decidability of pseudovarieties. In this context several concepts were introduced such as the notion of tameness, introduced by Almeida and Steinberg [7]. Tameness consists of a refinement of the concept of hyperdecidability previously introduced by Almeida [3].

\footnotetext{
${ }^{1}$ This work was supported, in part, by FCT through the Centro de Matemática da Universidade do Minho, and by the FCT and POCTI approved project POCTI/32817/MAT/2000 which is comparticipated by the European Community Fund FEDER.
} 
Tameness implies decidability but if it is preserved by the most common operators is an open problem. That it is a property of the "usual" pseudovarieties is a conjecture. In general, proving tameness of a pseudovariety is a non trivial, but useful, exercise. Some pseudovarieties are already known to be tame such as, for example: G (a consequence of the interpretation of Ash's results [10]); Ab [6], the pseudovariety of all finite abelian groups; $\mathbf{K}$ and $\mathbf{D}$ [9], the pseudovarieties of semigroups whose idempotents are, respectively, left zeros and right zeros. For more examples and recent developments on the subject, the reader is referred to Almeida's papers [4, 5].

Here we study the tameness of $\mathbf{L S 1}$, the pseudovariety of finite semigroups $S$ such that $e S e \in \mathbf{S l}$, for all idempotents $e \in S$. This problem is one of the concrete questions proposed by Almeida in [4]. Recall that the pseudovariety LSl is associated via Eilenberg's correspondence with the class of locally testable languages and that $\mathbf{L S l}=\mathbf{S} \mathbf{I} * \mathbf{D}[11,16]$. Notice that LSl is a subpseudovariety of $\mathbf{A}$. On the other hand, LSl is not contained in DS, the pseudovariety of semigroups whose regular $\mathcal{D}$-classes are semigroups, in contrast with most of the examples of pseudovarieties known to be tame. Proving the $\kappa$-tameness of LSl involves proving two properties: that the $\kappa$-word problem for LSl is decidable, and that LSl is $\kappa$-reducible. The first problem has already been solved by the first author in [12]. So, this paper is essentially dedicated to the proof of the second problem. We note that the problem of proving the $\kappa$-tameness of the semidirect product $\mathbf{S l} * \mathbf{K}$ is much more simple and was solved by the first author [13].

This work is organized as follows. After a section of preliminaries, where we introduce some notation and review some basic results on semigroups, pseudovarieties, words, graphs, $\kappa$-reducibility and $\kappa$-tameness, we present in Section 3 some properties about subpseudovarieties of $\mathbf{L S}$ l and their respective implicit operations. Section 4 is devoted to introduce some definitions on words and to establish some technical combinatoric results which are essential for our purposes. We next recall the result which establishes the decidability of the $\kappa$-word for LSl in Section 5. Finally, Section 6 gives the proof of the $\kappa$-reducibility of $\mathbf{L S l}$.

\section{Preliminaries}

For general background and terminology from the classical theories of semigroups, formal languages, pseudovarieties and profinite semigroups the reader is referred to $[2,8,17]$. Here, we begin by presenting a brief description of the most relevant basic definitions, notation and results that will be needed in the following sections.

\section{$2.1 \quad$ Words}

A finite non-empty set $A$ is called an alphabet and its elements are called letters. A (finite) word on $A$ is a finite sequence $w=\left(a_{1}, \ldots, a_{n}\right)$ of elements of $A$, usually written $w=a_{1} \cdots a_{n}$. The integer $n$ is called the length of $w$. The empty sequence, called the empty word, is denoted by 1 and its length is 0 . The length of a word $w$ is denoted by $|w|$. The product of two words $w=a_{1} a_{2} \cdots a_{n}$ and $z=b_{1} b_{2} \cdots b_{m}$ is the word $w z=a_{1} a_{2} \cdots a_{n} b_{1} b_{2} \cdots b_{m}$. We denote by $A^{*}$ the set of words on $A$ and by $A^{+}$the 
set of non-empty words. The set $A^{*}$ (resp. $A^{+}$) endowed with the product is a monoid (resp. semigroup) whose identity is the empty word and is called the free monoid (resp. the free semigroup) generated by $A$.

A word $w \in A^{+}$is said to be primitive if it is not a power of another word; that is, if $w=u^{n}$ for some $u \in A^{*}$ and $n \in \mathbb{N}$ implies $w=u$ (and $n=1$ ). Two words $w$ and $z$ are said to be conjugate if there exist words $u, v \in A^{*}$ such that $w=u v$ and $z=v u$. We notice that, if $w$ is a primitive word and $z$ is a conjugate of $w$, then $z$ is also primitive. Let an order be fixed for the letters of the alphabet A. A Lyndon word is a primitive word which is minimal, with respect to the lexicographic ordering, in its conjugation class.

A bi-infinite (resp. right-infinite, left-infinite) word on $A$ is a sequence $w=\left(a_{n}\right)_{n}$ of letters of $A$ indexed by $\mathbb{Z}$ (resp. $\mathbb{N},-\mathbb{N}$ ), also written

$$
w=\cdots a_{-2} a_{-1} a_{0} a_{1} a_{2} \cdots \quad\left(\text { resp. } w=a_{1} a_{2} \cdots, \quad w=\cdots a_{-2} a_{-1}\right) .
$$

We denote $w(n)=a_{n}$ and say that $a_{n}$ is the letter of $w$ on position $n$. For integers $i$ and $j$ such that $i<j$, we denote

$$
w\left[i, i\left[=1, \quad w\left[i, j\left[=a_{i} \cdots a_{j-1}, \quad w\left[i,+\infty\left[=a_{i} a_{i+1} \cdots .\right.\right.\right.\right.\right.\right.
$$

Analogously one would define $w] i, j], w[i, j], w]-\infty, j[$, etc. When they make sense, these notations are used also for finite and infinite words.

A word $x \in A^{*}$ is a factor of a word $w$, and $w$ is an extension of $x$, if $x=1$ or $x=w[i, j]$, for some integers $i$ and $j$. In such case, $w[i, j]$ is said to be an occurrence of the factor $x$ in $w$. We will denote by $\operatorname{Fact}(w)$ the set of all factors of $w$ and, for a positive integer $k$, by $\operatorname{Fact}_{k}(w)$ the set of all factors of $w$ of length $k$.

We say that two occurrences $w[i, j]$ and $w[k, l]$ of factors in a word $w$ are disjoint if the integer intervals $[i, j]$ and $[k, l]$ are disjoint sets. For each pair of words $w, x \in A^{*}$, we denote by $\operatorname{occ}(x, w)$ the number of occurrences of $x$ in $w$, and by $\operatorname{docc}(x, w)$ the maximal number of disjoint occurrences of $x$ in $w$. Of course, we have $\operatorname{docc}(x, w) \leq \operatorname{occ}(x, w)$. For instance, occ $($ baab, baabaaba $)=2$ and $\operatorname{docc}(b a a b, b a a b a a b a)=1$.

The sets of bi-infinite, right-infinite and left-infinite words on $A$ will be denoted, respectively, by $A^{\mathbb{Z}}, A^{\mathbb{N}}$ and $A^{-\mathbb{N}}$. We denote also

$$
A^{\infty}=A^{+} \cup A^{\mathbb{N}} \text { and } A^{-\infty}=A^{+} \cup A^{-\mathbb{N}} .
$$

The product of two elements $w$ and $z$ of $A^{\infty}$ is defined as follows: if $w, z \in A^{+}$, then $w z$ is already defined; right-infinite words are left zeros; finally, if $w=a_{0} a_{1} \cdots a_{n}$ is a finite word and $z=b_{1} b_{2} \cdots$ is a right-infinite word then $w z$ is the right-infinite word

$$
w z=a_{0} a_{1} \cdots a_{n} b_{1} b_{2} \cdots
$$

The product of two elements of $A^{-\infty}$ is defined symmetrically. Now, we observe that $A^{\mathbb{N}}, A^{-\mathbb{N}}, A^{\infty}$ and $A^{-\infty}$ are semigroups.

A word $x \in A^{*}$ is a prefix of a word $w \in A^{\infty}$, and $w$ is a right-extension of $x$, if there exists $z \in A^{\infty}$ such that $w=x z$. Symmetrically, $x \in A^{*}$ is a suffix of $w \in A^{-\infty}$, and $w$ is a left-extension of $x$, if there exists $z \in A^{-\infty}$ such that $w=z x$. 
A right-infinite word of the form $v u^{+\infty}=v u u u \cdots$, with $u \in A^{+}$and $v \in A^{*}$, is said to be ultimately periodic and $u$ is said to be a period of $v u^{+\infty}$. Dually, we will use the notation $u^{-\infty} v$ to represent the (ultimately periodic of period $u$ ) left-infinite word $u^{-\infty} v=\cdots u u u v$. As one can easily verify, a (right-infinite or left-infinite) ultimately periodic word has one and only one period which is a Lyndon word. An ultimately periodic word $w \in A^{\mathbb{N}}$ (resp. $w \in A^{-\mathbb{N}}$ ) which can be written in the form $w=u^{+\infty}$ (resp. $w=u^{-\infty}$ ) for some $u \in A^{+}$, is said to be periodic.

When writing a specific bi-infinite word, we need to specify which is the letter on position 0 . We do this by putting a "." on the left of the letter. For example,

$$
w=\cdots a a a \cdot b a a a \cdots
$$

represents the bi-infinite word over $\{a, b\}$ which contains exactly one occurrence of the letter $b$, being this occurrence on position 0 . This word can also be written $w=a^{-\infty} \cdot b a^{+\infty}$. More generally, given words $x=\left(x_{i}\right)_{i \in-\mathbb{N}} \in A^{-\mathbb{N}}$ and $y=\left(y_{i}\right)_{i \in \mathbb{N}} \in A^{\mathbb{N}}$, we denote by $x \cdot y$ the bi-infinite word $w=\cdots x_{-2} x_{-1} \cdot y_{1} y_{2} \cdots ;$ that is, $w$ is the bi-infinite word such that $w_{i}=x_{i}$ for $i \leq-1$ and $w_{i}=y_{i+1}$ for $i \geq 0$. A bi-infinite word $w$ is said to be ultimately periodic if $w=x \cdot y$ for some ultimately periodic words $x \in A^{-\mathbb{N}}$ and $y \in A^{\mathbb{N}}$. The word $w$ is said to be periodic if one can choose $x=u^{-\infty}$ and $y=u^{+\infty}$ for some $u \in A^{+}$. For instance, the word $w=a^{-\infty} \cdot b a^{+\infty}$ is ultimately periodic and non-periodic.

For each $n \in \mathbb{Z}$, we define a shift function $\sigma^{n}: A^{\mathbb{Z}} \rightarrow A^{\mathbb{Z}}$ by setting, for each $x=\left(x_{i}\right)_{i \in \mathbb{Z}} \in A^{\mathbb{Z}}, \sigma^{n}(x)=\left(x_{i+n}\right)_{i \in \mathbb{Z}}$. The element $\sigma^{n}(x)$ is called a shift of $x$. For instance, $\sigma^{3}\left(a^{-\infty} \cdot b a^{+\infty}\right)=a^{-\infty} b a a \cdot a^{+\infty}$. Notice that for $n>0$, the function $\sigma^{n}$ shifts letters $n$ places to the left, while $\sigma^{-n}$ shifts them $n$ places to the right. It is well known that a bi-infinite word $w$ is periodic if and only if $w=\sigma^{n}(w)$, for some $n>0$.

We denote by $\sim$ the equivalence relation on $A^{\mathbb{Z}}$ given by

$$
w \sim z \quad \text { if and only if } \exists n \in \mathbb{Z}, z=\sigma^{n}(w) .
$$

Given a left-infinite word $x \in A^{-\mathbb{N}}$ and a right-infinite word $y \in A^{\mathbb{N}}$, we denote by $x y$ the $\sim$-class of the bi-infinite word $x \cdot y$. For instance, $a^{-\infty} b a^{+\infty}$ is the $\sim$-class of all bi-infinite words over $\{a, b\}$ which contain exactly one occurrence of the letter $b$. For $u \in A^{+}$, we will usually denote $u^{\infty}=u^{-\infty} u^{+\infty}$. Sometimes we will not distinguish a bi-infinite word from its $\sim$-class.

\subsection{Semigroups, pseudovarieties and implicit operations}

If $S$ is a semigroup, we denote by $S^{1}$ the smallest monoid containing $S$. The set of idempotents of a semigroup $S$ is denoted by $E(S)$. A morphism of semigroups is a mapping $\varphi: S \rightarrow T$ between semigroups $S$ and $T$ that respects the multiplication operation of the semigroups. A relational morphism of semigroups is a binary relation $\theta: S \leftrightarrow T$ between semigroups $S$ and $T$ with domain $\mathrm{S}$ which is a subsemigroup of $S \times T$.

We consider finite sets endowed with discrete topology. Given an element $s$ of a compact semigroup, the smallest closed subsemigroup that contains $s$ has a unique 
idempotent, denoted by $s^{\omega}$. Moreover $s^{\omega-1}$ denotes the inverse of $s^{\omega+1}\left(=s^{\omega} s\right)$ in the maximal closed subgroup containing $s^{\omega}$.

A proof of the following classical result can be found in [2, Proposition 3.7.1].

Lemma 2.1 Let $S$ be a finite semigroup with $k$ elements. Then, for any $s_{1}, \ldots, s_{k} \in S$, there exist integers $1 \leq i \leq j \leq k$, such that, $s_{1} \cdots s_{k}=s_{1} \cdots s_{i-1}\left(s_{i} \cdots s_{j}\right)^{\omega} s_{j+1} \cdots s_{k}$.

A semigroup pseudovariety is a class of finite semigroups closed under taking subsemigroups, homomorphic images and finite direct products.

For a pseudovariety $\mathbf{V}$, a pro- $\mathbf{V}$ semigroup is a compact semigroup which is residually in $\mathbf{V}$. In case $\mathbf{V}=\mathbf{S}$, where $\mathbf{S}$ is the pseudovariety of all finite semigroups, we say that $S$ is profinite. The semigroup $\bar{\Omega}_{A} \mathbf{V}$ is a pro- $\mathbf{V}$ semigroup which is the free semigroup over a set $A$ for the class of all pro- $\mathbf{V}$ semigroups, which means that, for each pro- $\mathbf{V}$ semigroup $S$ and each continuous function $\varphi: A \longrightarrow S$, there is a unique continuous morphism $\bar{\varphi}: \bar{\Omega}_{A} \mathbf{V} \longrightarrow S$ extending $\varphi$. This leads to a natural interpretation of elements of $\bar{\Omega}_{A} \mathbf{V}$ as (A-ary) implicit operations: to each $\pi \in \bar{\Omega}_{A} \mathbf{V}$ is associated an operation $\pi_{S}: S^{A} \longrightarrow S$ which maps $\varphi \in S^{A}$ to $\pi \bar{\varphi}$. Recall that an implicit operation is a family of operations defined on the members of a pseudovariety $\mathbf{V}$ that commute with all homomorphisms between elements of $\mathbf{V}$. The subsemigroup of $\bar{\Omega}_{A} \mathbf{V}$ generated by $A$ is denoted by $\Omega_{A} \mathbf{V}$ and is a dense subsemigroup of $\bar{\Omega}_{A} \mathbf{V}$, whose elements are called explicit operations. The following examples of implicit operations play an important role in the next sections: the binary implicit operation multiplication, denoted by _. _ interpreted as the semigroup multiplication on each profinite semigroup, and the unary implicit operations $\omega$-power and $(\omega-1)$-power, denoted by ${ }_{-}{ }^{\omega}$ and ${ }_{-}{ }^{\omega-1}$, which, for a profinite semigroup $S$, to $s \in S$ associate $s^{\omega}$ and $s^{\omega-1}$, respectively. Note that the composition of implicit operations is an implicit operation.

We denote by $q_{\mathbf{V}}: \bar{\Omega}_{A} \mathbf{S} \longrightarrow \bar{\Omega}_{A} \mathbf{V}$ the continuous morphism mapping the generators of $\bar{\Omega}_{A} \mathbf{S}$ to the generators of $\bar{\Omega}_{A} \mathbf{V}$.

A pseudoidentity is a formal equality $\pi=\rho$ where $\pi, \rho \in \bar{\Omega}_{A} \mathbf{S}$ with $A$ finite. A finite semigroup $S$ satisfies a pseudoidentity $\pi=\rho$ if, for every continuous function $\varphi: A \longrightarrow S$, $\pi \bar{\varphi}=\rho \bar{\varphi}$. A pseudovariety $\mathbf{V}$ satisfies a pseudoidentity $\pi=\rho$ if every semigroup in $\mathbf{V}$ satisfies $\pi=\rho$, which means that $\pi q_{\mathbf{V}}=\rho q_{\mathbf{V}}$. Reiterman's theorem [18] states that every pseudovariety $\mathbf{V}$ is defined by a set $\Sigma$ of pseudoidentities, in the sense that $\mathbf{V}$ is the class of all finite semigroups that satisfy all the pseudoidentities of $\Sigma$, and in such case we write $\mathbf{V}=\llbracket \Sigma \rrbracket$.

Given $\pi \in \bar{\Omega}_{A} \mathbf{S}$, we say that $\rho \in \bar{\Omega}_{A} \mathbf{S}$ is a factor (resp. a prefix, a suffix) of $\pi$ if there are $\pi_{1}, \pi_{2} \in\left(\bar{\Omega}_{A} \mathbf{S}\right)^{1}$ such that $\pi=\pi_{1} \rho \pi_{2}$ (resp. $\pi=\rho \pi_{2}, \pi=\pi_{1} \rho$ ). A bi-infinite word $w$ is a (bi-infinite) factor of $\pi$ if every finite word which is a factor of $w$ is also a factor of $\pi$. Notice that, a bi-infinite word $w$ is a factor of $\pi$ if and only if every shift of $w$ is a factor of $\pi$. 


\subsection{Graphs}

In this paper all graphs considered are directed. So by a graph $\Gamma$ we mean a partial algebra with a support set $\mathcal{V}(\Gamma) \cup \mathcal{\cup}(\Gamma)$ with two sorts of elements, called vertices and edges respectively, and two unary operations $\alpha, \omega: \mathcal{E}(\Gamma) \rightarrow \mathcal{V}(\Gamma)$ which define the orientation of the edges. For any edge e $\in \mathcal{E}(\Gamma)$, e $\alpha$ is the beginning of e and e $\omega$ is the end of e.

Two edges $e_{1}$ and $e_{2}$ are said to be consecutive if $e_{1} \omega=e_{2} \alpha$. A path of $\Gamma$ is a finite sequence $e_{1}, e_{2}, \ldots, e_{n}$ of consecutive edges of $\Gamma$. Given a sequence of edges $e_{1}, \ldots, e_{n}$, if it is possible to invert the orientation of some edges in such a way to obtain a path, we say that $\mathrm{e}_{1}, \ldots, \mathrm{e}_{n}$ is a non-oriented path. A path $\mathrm{e}_{1}, \ldots, \mathrm{e}_{n}$ is a circuit if $\mathrm{e}_{1} \alpha=\mathrm{e}_{n} \omega$. By similarity, a non-oriented path is a non-oriented circuit if it is possible to invert the orientation of some edges in such a way to obtain a circuit.

A graph $\Gamma$ is said to be connected if for every pair of vertices of $\Gamma$ there is a nonoriented path between them. It is said to be strongly connected if, for any two of its vertices $v_{1}$ and $v_{2}$, there is a path from $v_{1}$ to $v_{2}$ and a path from $v_{2}$ to $v_{1}$. The connected component (resp. strongly connected component) of a vertex of $\Gamma$ is the largest connected subgraph (resp. strongly connected subgraph) of $\Gamma$ containing that vertex.

By a labelling of a graph $\Gamma$ by a semigroup $S$, we mean a mapping $\delta: \Gamma \longrightarrow S^{1}$ such that $\mathcal{E}(\Gamma) \delta \subseteq S$. Such labelling is consistent if $\mathrm{e} \alpha \delta \cdot \mathrm{e} \delta=\mathrm{e} \omega \delta$, for all $\mathrm{e} \in \mathcal{E}(\Gamma)$. Define the label of a path $\mathrm{e}_{1}, \ldots, \mathrm{e}_{n}$ to be $\mathrm{e}_{1} \delta \cdots \mathrm{e}_{n} \delta$. If $\gamma: \mathcal{E}(\Gamma) \longrightarrow A^{+}$is a function, the label of a non-oriented path $e_{1}, \ldots, e_{n}$ is the reduced form of the word $\left(e_{1} \gamma\right)^{\epsilon_{1}} \cdots\left(e_{n} \gamma\right)^{\epsilon_{n}}$ in the free group generated by $A$, where $\epsilon_{i}=1$ if in the non-oriented path the edge $\mathrm{e}_{i}$ is read in the direct way and $\epsilon_{i}=-1$ otherwise. We say that the function $\gamma$ commutes if the label of any non-oriented circuit is 1 .

\section{$2.4 \kappa$-reducibility and $\kappa$-tameness}

An implicit signature $\sigma$ is a set of implicit operations containing the multiplication. Every profinite semigroup can naturally be considered a topological algebra for the signature $\sigma$. Such algebras will be called $\sigma$-semigroups. For a pseudovariety $\mathbf{V}$, we denote by $\Omega_{A}^{\sigma} \mathbf{V}$ the free $\sigma$-semigroup generated by $A$ in the variety of $\sigma$-semigroups generated by $\mathbf{V}$, which as one can observe is the $\sigma$-subsemigroup of $\bar{\Omega}_{A} \mathbf{V}$ generated by $A$. The elements of $\Omega_{A}^{\sigma} \mathbf{S}$ are called $\sigma$-words or $\sigma$-terms. We denote by $p_{\mathbf{V}}: \Omega_{A}^{\sigma} \mathbf{S} \longrightarrow \Omega_{A}^{\sigma} \mathbf{V}$ the morphism of $\sigma$-semigroups determined by the choice of generators. That is, $p_{\mathbf{V}}$ is the restriction of $q_{\mathbf{V}}$ to $\Omega_{A}^{\sigma} \mathbf{S}$. The $\sigma$-word problem for $\mathbf{V}$ is the problem of deciding, for $\sigma$-words $\pi$ and $\rho$, if $\mathbf{V}$ satisfies the pseudoidentity $\pi=\rho$, that is, if $\pi p_{\mathbf{V}}=\rho p_{\mathbf{V}}$.

Let $\gamma: \Gamma \longrightarrow S^{1}$ be a labelling of a finite graph $\Gamma$ by a finite semigroup $S$ and let $\mu: S \rightarrow T$ be a relational morphism between semigroups $S$ and $T$. The labelling $\gamma$ is said to be $\mu$-inevitable if there is a consistent labelling $\delta: \Gamma \longrightarrow T^{1}$ such that $(\mathrm{g} \gamma, \mathrm{g} \delta) \in$ $\mu \cup\{(1,1)\}$ for every $\mathbf{g} \in \Gamma$. The labelling $\gamma$ is said to be $\mathbf{V}$-inevitable if it is $\mu$-inevitable for every relational morphism $\mu: S \longrightarrow T$, with $T \in \mathbf{V}$. Using a compactness result, Almeida [3] has shown the following result. 
Proposition 2.2 A labelling $\gamma$ of a finite graph $\Gamma$ by a finite A-generated semigroup $S$ is $\mathbf{V}$-inevitable if and only if there exists a labelling $\delta$ of $\Gamma$ by $\bar{\Omega}_{A} \mathbf{S}$ such that $\delta \psi=\gamma$, where the homomorphism $\psi: \bar{\Omega}_{A} \mathbf{S} \rightarrow S$ respects the choice of generators, and $\delta q_{\mathbf{V}}$ is consistent.

The pseudovariety $\mathbf{V}$ is said to be $\sigma$-reducible if every $\mathbf{V}$-inevitable labelling of a finite graph by a finite semigroup $S$ is $\mu_{\mathbf{V}^{-}}^{\sigma}$-inevitable, where $\mu_{\mathbf{V}}^{\sigma}=\varphi^{-1} p_{\mathbf{V}}$, for $\varphi: \Omega_{A}^{\sigma} \mathbf{S} \longrightarrow S$ the morphism of $\sigma$-semigroups which respects the choice of generators.

The following commutative diagram expresses the $\sigma$-reducibility of $\mathbf{V}$, which means that proving $\sigma$-reducibility of $\mathbf{V}$ is proving the existence of $\delta^{\prime}$ in the conditions of the diagram.

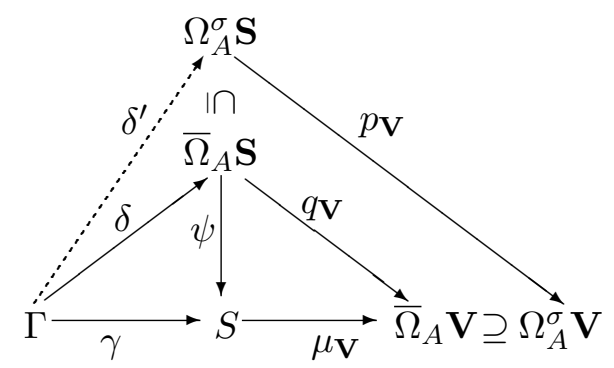

A pseudovariety $\mathbf{V}$ is said to be $\sigma$-tame if it is recursively enumerable, $\sigma$-reducible and the $\sigma$-word problem for $\mathbf{V}$ is decidable. Finally we say that a pseudovariety is tame if it is $\sigma$-tame with respect to a recursively enumerable implicit signature $\sigma$ consisting of computable implicit operations.

In this paper, we are going to consider the implicit signature $\kappa=\left\{{ }_{-},_{-}{ }^{\omega}\right\}$. The most commonly used signature is $\left\{{ }_{-} \cdot,_{-}{ }^{\omega-1}\right\}$ but, since we are going to work with aperiodic semigroups and $\mathbf{A}$ satisfies $x^{\omega}=x^{\omega-1}$, we will use $\kappa$.

\section{Implicit operations on LSl}

This section gathers some basic statements about the free pro- $\mathbf{V}$ semigroups, when $\mathbf{V} \in\{\mathbf{K}, \mathbf{D}, \mathbf{L I}, \mathbf{L S l}\}$ which will be used in this work. Recall that $\mathbf{K}, \mathbf{D} \subseteq \mathbf{L I} \subseteq \mathbf{L S l}$,

$$
\mathbf{K}=\llbracket x^{\omega} y=x^{\omega} \rrbracket, \mathbf{D}=\llbracket y x^{\omega}=x^{\omega} \rrbracket, \quad \mathbf{L I}=\llbracket x^{\omega} y z^{\omega}=x^{\omega} z^{\omega} \rrbracket
$$

and

$$
\mathbf{L S} \mathbf{l}=\llbracket x^{\omega} y x^{\omega} y x^{\omega}=x^{\omega} y x^{\omega}, x^{\omega} y x^{\omega} z x^{\omega}=x^{\omega} z x^{\omega} y x^{\omega} \rrbracket .
$$

Notice also that $\mathbf{K} \vee \mathbf{D}=\mathbf{L I}$. This means that a pseudoidentity $\pi=\rho$ is satisfied by $\mathbf{L I}$ if and only if it is satisfied by both $\mathbf{K}$ and $\mathbf{D}$. The following properties are well known (see [2], for instance).

Lemma 3.1 Let $\mathbf{V}$ be one of the pseudovarieties $\mathbf{K}, \mathbf{D}$ or $\mathbf{L I}$. Then, $\Omega_{A} \mathbf{V}$ is isomorphic to $A^{+}, E\left(\bar{\Omega}_{A} \mathbf{V}\right)$ is an ideal consisting of the non-explicit operations of $\bar{\Omega}_{A} \mathbf{V}$ and $\bar{\Omega}_{A} \mathbf{K}$, $\bar{\Omega}_{A} \mathbf{D}$ and $E\left(\bar{\Omega}_{A} \mathbf{L I}\right)$ are isomorphic to, respectively, $A^{\infty}, A^{-\infty}$ and $A^{\mathbb{N}} \times A^{-\mathbb{N}}$. 
In view of this lemma, we shall identify explicit operations (on $\mathbf{K}, \mathbf{D}$ or $\mathbf{L I}$ ) with finite words. Moreover, the idempotents of $\bar{\Omega}_{A} \mathbf{K}$ and $\bar{\Omega}_{A} \mathbf{D}$ may be identified, respectively, with right-infinite words and left-infinite words. Notice that in $\bar{\Omega}_{A} \mathbf{K}$, the right-infinite word $y x^{+\infty}$, where $y \in A^{*}$ and $x \in A^{+}$, corresponds to the implicit operation $y x^{\omega}$. A dual remark holds for $\mathbf{D}$.

Let $n$ be a positive integer. We denote by $\equiv_{n}$ the congruence on $\bar{\Omega}_{A} \mathbf{S}$ given, for every $\pi, \rho \in \bar{\Omega}_{A} \mathbf{S}$, by

$$
\pi \equiv_{n} \rho \quad \text { if } \quad \pi \text { and } \rho \text { have the same prefix, suffix and factors of length } n \text {. }
$$

We summarize in the next proposition some properties of the pseudoidentities satisfied by LSl. This result is an immediate consequence of Theorem 3.3 in [12].

Proposition 3.2 Let $\pi, \rho \in \bar{\Omega}_{A} \mathbf{S}$. The following conditions are equivalent:

i) $\pi q_{\mathbf{L S l}}=\rho q_{\mathbf{L S l}}$;

ii) $\pi \equiv_{n} \rho$ for every $n \in \mathbb{N}$;

iii) $\pi q_{\mathbf{L I}}=\rho q_{\mathbf{L I}}$ and $\pi$ and $\rho$ have the same finite factors;

iv) $\pi q_{\mathbf{L I}}=\rho q_{\mathbf{L I}}$ and $\pi$ and $\rho$ have the same bi-infinite factors.

Moreover, if $\pi$ and $\rho$ are non-explicit, then a bi-infinite word $\mathbf{w} \in A^{\mathbb{Z}}$ is a factor of $\pi \rho$ if and only if $\mathbf{w}$ is a factor of $\pi$ or a factor of $\rho$, or $\mathbf{w}$ is a shift of the bi-infinite word $\pi q_{\mathbf{D}} \cdot \rho q_{\mathbf{K}}$.

Now we state the following simple, but fundamental, result.

Lemma 3.3 Let $S \in \mathbf{S}, \pi \in \bar{\Omega}_{A} \mathbf{S}$ be a non-explicit operation and $n \in \mathbb{N}$. Then, there exists a word $u_{\pi} \in A^{+}$such that $u_{\pi} \equiv_{n} \pi$ and $S$ satisfies $\pi=u_{\pi}$.

Proof. Let $\left(u_{i}\right)_{i}$ be a sequence of words converging to $\pi$. Since $S$ is finite, $S$ satisfies $u_{i}=\pi$ for every $i$ after a certain index. Moreover, taking $i$ sufficiently large, the word $u_{\pi}=u_{i}$ is such that $u_{\pi} \equiv_{n} \pi$ and $S$ satisfies $\pi=u_{\pi}$.

A proof of the following useful result can be found in [9].

Lemma 3.4 Let $\pi, \rho \in \bar{\Omega}_{A} \mathbf{S}$ be non-explicit operations such that $\pi q_{\mathbf{D}}=\rho q_{\mathbf{D}}$. Then there exist factorizations $\pi=\pi_{1} \pi_{2}$ and $\rho=\rho_{1} \pi_{2}$ where $\pi_{1}, \pi_{2}, \rho_{1}$ are non-explicit operations. Moreover, if $\pi q_{\mathbf{D}}=u^{-\infty} v$ where $u \in A^{+}$and $v \in A^{*}$, then one can choose $\pi_{2}=u^{\omega} v$.

A dual result could be stated for the pseudovariety $\mathbf{K}$.

\section{Some combinatorial results}

In this section, we introduce some definitions on words and prove some results that will be used later. 


\subsection{Centers of bi-infinite words}

Let $u \in A^{+}$and let $x=u[l, r]$ be an occurrence of a factor $x$ in $u$. An occurrence $v=u\left[l^{\prime}, r\right]$, with $l^{\prime} \leq l$, of a factor $v$ in $u$ is said to be a left-extension of the occurrence $u[l, r]$. In this case, the word $v$ itself is said to be a left-extension (in $u$ ) of the occurrence $u[l, r]$.

Definition 4.1 (allowed occurrence) Let $x \in A^{+}$and let $\overleftarrow{x} \in A^{+}$be a left-extension of $x$. We say that an occurrence $x=u[l, r]$ in a word $u \in A^{+}$is allowed in $u$ relatively to $\overleftarrow{x}$ if $\overleftarrow{x}$ is a left-extension in $u$ of the occurrence $u[l, r]$

For instance, let $x=a b$ and let $\overleftarrow{x}=a a b a b$. Then

$$
u=b a a b \boldsymbol{a b} a b a a b \boldsymbol{a b}
$$

has two allowed occurrences of $x: u[5,6]$ and $u[12,13]$; and three occurrences of $x$ not allowed: $u[3,4], u[7,8]$ and $u[10,11]$. If $\overleftarrow{x}=b b a b$ then $x$ has no allowed occurrences in $u$.

Let $\mathbf{w} \in A^{\mathbb{Z}}$ be a bi-infinite word. For every pair of integers $p, q \in \mathbb{N}_{0}(=\mathbb{N} \cup\{0\})$, the factor $\mathbf{w}[-p, q]$ is said to be a center of $\mathbf{w}$. Let $\mathbf{w}^{\prime} \in A^{\mathbb{Z}}$ be another bi-infinite word such that $\mathbf{w} \nsim \mathbf{w}^{\prime}$ (i.e., $\mathbf{w}^{\prime}$ is not a shift of $\mathbf{w}$ ). An occurrence of $\mathbf{w}[-p, q]$ in $\mathbf{w}^{\prime}$, when it exists, is of the form $\mathbf{w}^{\prime}[h-p, h+q]$ for some $h \in \mathbb{Z}$. Notice that in this case $h$ is the position in $\mathbf{w}^{\prime}$ of the central letter $\mathbf{w}(0)$ of $\mathbf{w}$. We then say that the center $\mathbf{w}[-p, q]$ of $\mathbf{w}$ occurs in $\mathbf{w}^{\prime}$ on position $h$. Notice that, since by hypothesis $\mathbf{w} \nsim \mathbf{w}^{\prime}$, there is not a $h \in \mathbb{N}_{0}$ such that $\mathbf{w}[-p, q]$ occurs in $\mathbf{w}^{\prime}$ on position $h$, for every $p$ and $q$.

We now assume that $\mathbf{w}$ and $\mathbf{w}^{\prime}$ are non-periodic and prove the following statement.

$\exists q \in \mathbb{N}_{0} \forall h \in \mathbb{N}_{0} \exists p \in \mathbb{N}_{0}, \mathbf{w}[-p, q]$ does not occur in $\mathbf{w}^{\prime}$ on a position in $[-h, h]$

Suppose that (1) is not true. Hence,

$$
\forall q \in \mathbb{N}_{0} \exists h \in \mathbb{N}_{0} \forall p \in \mathbb{N}_{0}, \mathbf{w}[-p, q] \text { occurs in } \mathbf{w}^{\prime} \text { on a position in }[-h, h] .
$$

Since the integer interval $[-h, h]$ is finite, we deduce that

$$
\forall q \in \mathbb{N}_{0} \exists h_{q} \in \mathbb{Z} \forall p \in \mathbb{N}_{0}, \mathbf{w}[-p, q] \text { occurs in } \mathbf{w}^{\prime} \text { on position } h_{q} .
$$

Let $q \in \mathbb{N}_{0}$. Then, for every integer $i \leq q, \mathbf{w}(i)=\mathbf{w}^{\prime}\left(i+h_{q}\right)$, so that

$$
\left.\left.\mathbf{w}]-\infty, q]=\mathbf{w}^{\prime}\right]-\infty, q+h_{q}\right]
$$

Since $\mathbf{w} \not \mathbf{w}^{\prime}$, the equality $h_{q}=h_{q^{\prime}}$ holds only for a finite number of integers $q^{\prime} \in \mathbb{N}_{0}$. Therefore, $h_{q} \neq h_{q^{\prime}}$ for some integer $q^{\prime}>q$. On the other hand $\left.\left.\left.\mathbf{w}\right]-\infty, q^{\prime}\right]=\mathbf{w}^{\prime}\right]-$ $\left.\infty, q^{\prime}+h_{q^{\prime}}\right]$, whence $\left.\left.\left.\left.\left.\left.\mathbf{w}\right]-\infty, q\right]=\mathbf{w}^{\prime}\right]-\infty, q+h_{q}\right]=\mathbf{w}^{\prime}\right]-\infty, q+h_{q^{\prime}}\right]$. Assuming, without loss of generality, that $h_{q}<h_{q^{\prime}}$ and putting $\left.\left.u=\mathbf{w}^{\prime}\right] h_{q}, h_{q^{\prime}}\right]$, we deduce that $\mathbf{w}]-\infty, q]=u^{-\infty}$. Since $q$ is arbitrary, this clearly implies that $\mathbf{w}$ is periodic. This contradicts the hypothesis and so we conclude that (1) holds. 
Now, using the fact that $\mathbf{w}[-P, Q]$ is a factor of $\mathbf{w}[-p, q]$ for every $q \geq Q$ and $p \geq P$ and using symmetry, we easily deduce the more generic statement,

$$
\begin{array}{r}
\exists Q \in \mathbb{N}_{0} \forall q \geq Q \forall h \in \mathbb{N}_{0} \exists P \in \mathbb{N}_{0} \forall p \geq P, \mathbf{w}[-p, q] \text { does not occur in } \mathbf{w}^{\prime} \text { and } \\
\mathbf{w}^{\prime}[-p, q] \text { does not occur in } \mathbf{w} \text { on positions in the interval }[-h, h] .
\end{array}
$$

Fix two integers $q, q^{\prime} \geq Q$ and write $c_{i}=\mathbf{w}[-i, q]$ and $c_{i}^{\prime}=\mathbf{w}^{\prime}\left[-i, q^{\prime}\right]$ for every $i \in \mathbb{N}_{0}$. Let $t$ and $t^{\prime}$ be two positive integers. Then, by (2), for $h=\max \left(t, t^{\prime}\right)+\max \left(q, q^{\prime}\right)$ there exists $P \in \mathbb{N}_{0}$ such that

$$
\begin{gathered}
\forall p \geq P, \quad c_{p} \text { does not occur in } \mathbf{w}^{\prime} \text { and } c_{p}^{\prime} \text { does not occur in } \mathbf{w} \\
\text { on positions in the interval }[-h, h] .
\end{gathered}
$$

Let $p \geq \max \left(P, t, t^{\prime}\right)$, let $T$ be a large integer (to be more precise, it suffices to take $\left.T \geq \max \left(q, q^{\prime}\right)+2 p\right)$, and let $\overleftarrow{c_{t}}=c_{T}$ and $\overleftarrow{c_{t^{\prime}}^{\prime}}=c_{T}^{\prime}$. Our objective is to prove the following:

Claim Any two allowed occurrences of $c_{t}$ and $c_{t^{\prime}}^{\prime}$ in a finite word $u \in A^{+}$, relatively to $\overleftarrow{c_{t}}=c_{T}$ and $\overleftarrow{c_{t^{\prime}}^{\prime}}=c_{T}^{\prime}$ respectively, are disjoint

Indeed, suppose that $c_{t}=u[r-t, r+q]$ and $c_{t^{\prime}}^{\prime}=u\left[r^{\prime}-t^{\prime}, r^{\prime}+q^{\prime}\right]$ are two allowed occurrences in $u$. Since these occurrences are allowed, they can be extended, respectively, to occurrences $c_{T}=u[r-T, r+q]$ and $c_{T}^{\prime}=u\left[r^{\prime}-T, r^{\prime}+q^{\prime}\right]$ of $c_{T}=\overleftarrow{c_{t}}$ and $c_{T}^{\prime}=\overleftarrow{c_{t^{\prime}}^{\prime}}$ in $u$. Suppose without loss of generality that $r+q \leq r^{\prime}+q^{\prime}$ and that the occurrences $c_{t}=u[r-t, r+q]$ and $c_{t^{\prime}}^{\prime}=u\left[r^{\prime}-t^{\prime}, r^{\prime}+q^{\prime}\right]$ are not disjoint. Then $r-r^{\prime} \leq q^{\prime}-q$ and $r+q \geq r^{\prime}-t^{\prime}$. Hence $-q-t^{\prime} \leq r-r^{\prime} \leq q^{\prime}-q$ and we deduce from the definitions of $p$ and $T$ that

$$
r-p \geq r^{\prime}-t^{\prime}-q-p \geq r^{\prime}-q-2 p \geq r^{\prime}-T .
$$

Therefore, the occurrence $c_{p}=u[r-p, r+q]$ is contained in the occurrence $c_{T}^{\prime}=$ $u\left[r^{\prime}-T, r^{\prime}+q^{\prime}\right]$. Since $r-r^{\prime} \in\left[-q-t^{\prime}, q^{\prime}-q\right] \subseteq[-h, h]$, this implies that $c_{p}$ occurs in $\mathbf{w}^{\prime}$ on a position in the interval $[-h, h]$, which contradicts (3). Therefore, the occurrences $c_{t}=u[r-t, r+q]$ and $c_{t^{\prime}}^{\prime}=u\left[r^{\prime}-t^{\prime}, r^{\prime}+q^{\prime}\right]$ are disjoint, and the claim is true for every sufficiently large $T$.

Notice that, since $\mathbf{w} \in A^{\mathbb{Z}}$ is non-periodic (so that $\mathbf{w} \neq \sigma^{m}(\mathbf{w})$ for every integer $m \neq 0$ ), the claim remains true for $\mathbf{w}^{\prime}=\mathbf{w}$. That is, any two distinct allowed occurrences of $c_{t}=\mathbf{w}[-t, q]$ in a finite word $u$, relatively to $\overleftarrow{c_{t}}=c_{T}$, are disjoint

Since $T$ is arbitrarily large, we could consider any finite set of bi-infinite words instead of only two and deduce the following result.

Lemma 4.2 Let $B$ be a finite set of bi-infinite words and let, for each $\mathbf{w} \in B, t_{\mathbf{w}}$ be a positive integer. There exists a positive integer $Q$ such that, given an integer $q_{\mathbf{w}} \geq Q$ for each $\mathbf{w} \in B$, for every large integer $T \geq \max \left\{t_{\mathbf{w}} \mid \mathbf{w} \in B\right\}$, if $\mathbf{w}, \mathbf{w}^{\prime} \in B$ with $\mathbf{w} \neq \sigma^{m}\left(\mathbf{w}^{\prime}\right)$ for every integer $m \neq 0$ and there are two distinct occurrences of $\mathbf{w}\left[-t_{\mathbf{w}}, q_{\mathbf{w}}\right]$ and $\mathbf{w}\left[-t_{\mathbf{w}^{\prime}}, q_{\mathbf{w}^{\prime}}\right]$ which are allowed in a finite word $u \in A^{+}$, relatively to $\mathbf{w}\left[-T, q_{\mathbf{w}}\right]$ and $\mathbf{w}\left[-T, q_{\mathbf{w}^{\prime}}\right]$ respectively, then these occurrences of $\mathbf{w}\left[-t_{\mathbf{w}}, q_{\mathbf{w}}\right]$ and $\mathbf{w}\left[-t_{\mathbf{w}^{\prime}}, q_{\mathbf{w}^{\prime}}\right]$ are disjoint. 


\subsection{Marked factors}

In this paper, we will need to fix and use several integers which depend one on the others. For our and the readers convenience, we will introduce now some of these constants.

Definition 4.3 (constants $k, k^{\prime}$ and $\left.k^{\prime \prime}\right)$ We let:

- $k$ represent the number $|S|$ of elements of a finite A-generated semigroup $S$ which will be introduced in the beginning of Section 6;

- $k^{\prime}=6 k|A|^{3 k} ;$ (Notice that this constant is large enough to guarantee that, if $x_{1}, \ldots, x_{r}$ are all the factors of a word $u \in A^{+}$with $2 k \leq\left|x_{i}\right|<3 k$ and if $\operatorname{docc}\left(x_{i}, u\right)>k^{\prime}-2$ for every $1 \leq i \leq r$, then it is possible to choose one occurrence of each factor $x_{i}$ such that these occurrences are pairwise disjoint. We will be interested in these factors $x_{i}$ because they can be factored in the form $x_{i}=x_{i, 1} x_{i, 2} x_{i, 3}$ with $\left|x_{i, 1}\right|=\left|x_{i, 3}\right|=k$ and $\left|x_{i, 2}\right|<k$.)

- $k^{\prime \prime}=\left[2 k^{\prime}(3 k-1)\right]^{|A|^{3 k-1}+1}$. (The definition of $k^{\prime \prime}$ is motivated by Lemma 4.6 below.)

The definitions that follow, although given for the constants $k, k^{\prime}$ and $k^{\prime \prime}$ above, could be made for generic integers.

A finite word $v$ is said to be $k^{\prime}$-abundant if $\operatorname{docc}(y, v) \geq k^{\prime}$ for all factors $y$ of $v$ with length $3 k-1$.

Let $u=a_{1} a_{2} \cdots a_{n}\left(a_{i} \in A\right)$ be a finite word. A $k^{\prime \prime}$-neighborhood of an occurrence $x=u[i, j]$ of a factor $x$ in $u$ is an occurrence $v=u\left[i^{\prime}, j^{\prime}\right]$ extending $u[i, j]$ (i.e., such that $i^{\prime} \leq i$ and $j^{\prime} \geq j$ ) and such that $|v| \leq k^{\prime \prime}$. An occurrence $x=u[i, j]$ of a factor $x$ of length $3 k-1$ in $u$ will be said to be free if there exists a $k^{\prime \prime}$-neighborhood $v$ of $u[i, j]$ such that $v$ is $k^{\prime}$-abundant. Notice that, in this case, every occurrence of a factor $y$, of length $3 k-1$, in the $k^{\prime \prime}$-neighborhood $v$ is free. The occurrence $x=u[i, j]$ (and the letters $\left.a_{i}, a_{i+1}, \ldots, a_{j}\right)$ will be said to be marked if $u[i, j]$ is not free.

The next lemma follows easily from the above definitions.

Lemma 4.4 There is a unique factorization

$$
u=x_{0} w_{1} x_{1} w_{2} \cdots w_{r} x_{r}
$$

such that: if $|u|<3 k-1, u=w_{1}$; otherwise,

- $r \geq 0$;

- $x_{0}, x_{r} \in A^{*}, x_{1}, \ldots, x_{r-1} \in A^{+}$;

- for each $1 \leq i \leq r$, the letters of $w_{i}$ are marked;

- for each $0 \leq j \leq r$, the letters of $x_{j}$ are not marked.

Definition 4.5 (marked factorization) The factorization of Lemma 4.4 is called the marked factorization of $u$ (for $k$ ). The factors $w_{1}, \ldots, w_{r}\left(\right.$ resp $\left.. x_{0}, \ldots, x_{r}\right)$ are said to be the marked factors (resp. the free factors) of u (for $k$ ). 
In the following lemma, we prove that the marked factors of a word are of bounded length.

Lemma 4.6 Let $u=x_{0} w_{1} x_{1} w_{2} \cdots w_{r} x_{r}$ be the marked factorization of a word $u \in A^{+}$. Then $\left|w_{i}\right|<k^{\prime \prime}$ for every $1 \leq i \leq r$.

Proof. The lemma is obvious when $|u|<3 k-1$. So, we assume that $|u| \geq 3 k-1$. Let $b=2 k^{\prime}(3 k-1)$ and $e=|A|^{3 k-1}+1$, so that $k^{\prime \prime}=b^{e}$. Suppose that $\left|w_{i}\right| \geq k^{\prime \prime}$ for some integer $1 \leq i \leq r$. Then, since every letter of $w_{i}$ is marked, there is a marked occurrence $x_{1}$ of a factor of length $3 k-1$ in $w_{i}$. Let $v_{1}$ be an occurrence of length $k^{\prime \prime}$ in $w_{i}$ such that $v_{1}$ is a $k^{\prime \prime}$-neighborhood of $x_{1}$. In particular, since $x_{1}$ is marked, $v_{1}$ is not $k^{\prime}$-abundant. Hence,

$$
\exists y_{1} \in \operatorname{Fact}_{3 k-1}\left(v_{1}\right), \operatorname{docc}\left(y_{1}, v_{1}\right)<k^{\prime} .
$$

Notice that, therefore, the occurrences of $y_{1}$ in $v_{1}$ "use" less than $b$ letters; that is, if

$$
I=\bigcup_{\substack{[i, j] \subset \mathbb{N} \\ y_{1}=v_{1}[i, j]}}[i, j]
$$

then $\# I<b$. Hence, as $\left|v_{1}\right|=k^{\prime \prime}=b^{e}$, there is an integer interval $[h, g] \subseteq\left[1,\left|v_{1}\right|\right]$, of amplitude $b^{e-1}$, that does not contain any element of $I$. In particular, this implies that

$$
\exists v_{2} \in \operatorname{Fact}\left(v_{1}\right), \operatorname{occ}\left(y_{1}, v_{2}\right)=0 \text { and }\left|v_{2}\right|=b^{e-1} .
$$

Now, since $\left|v_{2}\right| \geq 2(3 k-1)$ and every letter of $v_{2}$ is marked, there is in $v_{2}$ an occurrence $x_{2}$ of length $3 k-1$ that is marked in $u$. In particular $v_{2}$ is a $k^{\prime \prime}$-neighborhood of $x_{2}$, whence $v_{2}$ is not $k^{\prime}$-abundant. Hence,

$$
\exists y_{2} \in \operatorname{Fact}_{3 k-1}\left(v_{2}\right), \operatorname{docc}\left(y_{2}, v_{2}\right)<k^{\prime} .
$$

Therefore, as above, one can show that

$$
\exists v_{3} \in \operatorname{Fact}\left(v_{2}\right), \operatorname{occ}\left(y_{2}, v_{3}\right)=0 \text { and }\left|v_{3}\right|=b^{e-2} .
$$

Notice that, as $v_{3}$ is a factor of $v_{2}, y_{1}$ does not occur in $v_{3}$. Therefore $v_{3}$ is a factor of $w_{i}$ of length $b^{e-2}$ with no occurrences of $y_{1}$ and $y_{2}$.

Iterating this process, one deduces, after $e-1=|A|^{3 k-1}$ steps, the existence of distinct words $y_{1}, \ldots, y_{e-1} \in A^{+}$of length $3 k-1$ and a factor $v_{e}$ of $w_{i}$ such that

$$
y_{1}, \ldots, y_{e-1} \notin \operatorname{Fact}\left(v_{e}\right) \text { and }\left|v_{e}\right|=b .
$$

Hence $\left|v_{e}\right| \geq 3 k-1$ and so $v_{e}$ has a factor of length $3 k-1$. This is absurd because $y_{1}, \ldots, y_{e-1}$ are $e-1$ distinct words on $A$ of length $3 k-1$ that are not factors of $v_{e}$ and there exist exactly $e-1$ distinct words on $A$ of length $3 k-1$. Therefore $\left|w_{i}\right|<k^{\prime \prime}$.

The process of marking letters of a given word $u \in A^{+}$, described in this subsection, is a way to localize the factors of a given length $3 k-1$ that (locally) have "few" occurrences: the definition of what "few" means is made in such a way that the marked factors have bounded lengths. 


\section{The $\kappa$-word problem for LSl}

A non-explicit $\kappa$-term $\pi$ of the form

$$
\pi=u_{0} x_{1}^{\omega} u_{1} x_{2}^{\omega} \cdots x_{n}^{\omega} u_{n}
$$

with $n \geq 1, u_{0}, \ldots, u_{n} \in A^{*}$ and $x_{1}, \ldots, x_{n} \in A^{+}$, will be called a rank $1 \kappa$-term. It is clear that $\pi q_{\mathbf{K}}$ is the right-infinite word $u_{0} x_{1}^{+\infty}$ and $\pi q_{\mathbf{D}}$ is the left-infinite word $x_{n}^{-\infty} u_{n}$. The bi-infinite words of the form $x_{i}^{\infty}$ and $x_{j}^{-\infty} u_{j} x_{j+1}^{+\infty}$, with $i=1, \ldots, n$ and $j=1, \ldots, n-1$, are the bi-infinite factors of $\pi$.

The $\kappa$-word problem for LSl is decidable as shown by the first author [12]. Note that, by Proposition 3.2, $\Omega_{A} \mathbf{L S l}$ is isomorphic to $A^{+}$. Moreover, if $\pi \in \bar{\Omega}_{A} \mathbf{S}$ is a nonexplicit operation and a pseudoidentity $\pi=\rho$ holds in LSl, then $\rho$ is non-explicit too. The decision criterion to test if two non-explicit $\kappa$-terms are equal over LSl is given in next proposition (Theorem 7.1 of [12]).

Proposition 5.1 Let $\pi \in \Omega_{A}^{\kappa} \mathbf{S}$ be a non-explicit $\kappa$-term. Then, there is a rank $1 \kappa$-term $\pi_{1}=u_{0} x_{1}^{\omega} u_{1} x_{2}^{\omega} \cdots x_{n}^{\omega} u_{n}$ such that $\pi q_{\mathbf{L S} \mathbf{I}}=\pi_{1} q_{\mathbf{L S} \mathbf{l}}$.

Moreover, if $\rho \in \Omega_{A}^{\kappa} \mathbf{S}$ is another non-explicit $\kappa$-term and $\rho_{1}=v_{0} y_{1}^{\omega} v_{1} y_{2}^{\omega} \cdots y_{m}^{\omega} v_{m}$ is a rank $1 \kappa$-term such that $\rho q_{\mathbf{L S} \mathbf{I}}=\rho_{1} q_{\mathbf{L S} \mathbf{l}}$, then $\pi q_{\mathbf{L S} \mathbf{I}}=\rho q_{\mathbf{L S}}$ if and only if $u_{0} x_{1}^{+\infty}=v_{0} y_{1}^{+\infty}$, $x_{n}^{-\infty} u_{n}=y_{m}^{-\infty} v_{m}$ and $\pi_{1}$ and $\rho_{1}$ have the same bi-infinite factors.

Furthermore, the equality $\pi q_{\mathbf{L S I}}=\rho q_{\mathbf{L S I}}$ is effectively decidable.

\section{$6 \kappa$-reducibility of LSl}

In this section, we show that the pseudovariety $\mathbf{L S I}$ is $\kappa$-reducible.

Let $\gamma$ be a LSl-inevitable labelling of a finite graph $\Gamma$ by a finite $A$-generated semigroup $S$ and let $\psi$ be the unique homomorphism from $\bar{\Omega}_{A} \mathbf{S}$ to $S$ which respects the choice of generators. By Proposition 2.2, there is a labelling $\delta$ of $\Gamma$ by $\bar{\Omega}_{A} \mathbf{S}$ such that $\delta \psi=\gamma$ and $\delta q_{\mathbf{L S} \mathbf{l}}$ is consistent. Notice that since $\mathbf{K}$ and $\mathbf{D}$ are subpseudovarieties of $\mathbf{L S l}$, this implies that $\delta q_{\mathbf{K}}$ and $\delta q_{\mathbf{D}}$ are both consistent. We have to construct a labelling $\delta^{\prime}: \Gamma \rightarrow \Omega_{A}^{\kappa} \mathbf{S}$ such that $\delta^{\prime} \psi=\gamma$ and $\delta^{\prime} p_{\mathbf{L S I}}$ is consistent.

\subsection{Reductions on the graph $\Gamma$}

We first reduce to the case in which all edges are labelled by non-explicit operations under $\delta$. Let $\mathcal{V}_{\omega}$ be the set of vertices $v \in \mathcal{V}(\Gamma)$ such that $\mathrm{v} \delta$ is a non-explicit operation. Let $\phi$ be the equivalence relation on $\mathcal{V}_{\omega}$ generated by the relation

$\left\{(\mathrm{v}, \mathrm{w}) \mid \mathrm{v}, \mathrm{w} \in \mathcal{V}_{\omega}\right.$ and there is an edge e from $\mathrm{v}$ to $\mathrm{w}$ such that $\mathrm{e} \delta$ is explicit $\}$.

For each $\vee \in \mathcal{V}_{\omega}$, let

- $\mathrm{v} \phi$ be the $\phi$-class of $\mathrm{v}$;

- $\mathcal{E}_{\mathrm{v} \phi}$ be the subset of $\mathcal{E}(\Gamma)$ consisting of all edges e such that e $\delta$ is explicit and $\mathrm{e} \omega \in \mathrm{v} \phi$ (and so also e $\alpha \in \mathrm{v} \phi)$; 
- $\mathcal{E}_{\mathrm{v}}$ be the set of edges e $\in \mathcal{E}(\Gamma)$ such that e $\omega=\mathrm{v}$;

- $\mathcal{E}_{\mathrm{v}, \omega}$ be the subset of $\mathcal{E}_{\mathrm{v}}$ consisting of all edges labelled under $\delta$ by a non-explicit operation;

- $\mathcal{E}_{\mathrm{v}, 0}=\mathcal{E}_{\mathrm{v}} \backslash \mathcal{E}_{\mathrm{v}, \omega}$.

Select a vertex $v_{0} \in \mathcal{V}_{\omega}$ such that $\mathcal{E}_{\mathrm{v}_{0} \phi}$ is non-empty. Let $m_{\mathrm{v}_{0} \phi}$ be the maximal length of labels of non-oriented paths, having no repeated edges, consisting of edges of $\mathcal{E}_{\mathrm{v}_{0} \phi}$. Since $\mathrm{v}_{0} \delta$ is not explicit, then, by Lemma 3.1 , there is a factorization

$$
\mathrm{v}_{0} \delta q_{\mathbf{D}}=z_{\mathrm{v}_{0} \phi} s_{\mathrm{v}_{0}}
$$

where $s_{\mathrm{v}_{0}}$ is a word of length $m_{\mathrm{v}_{0} \phi}$ and $z_{\mathrm{v}_{0} \phi} \in \bar{\Omega}_{A} \mathbf{D}$ is a non-explicit operation. Let $v \in v_{0} \phi$. Select a non-oriented path from $v_{0}$ to $v$ with no repeated edges and consisting of edges of $\mathcal{E}_{\mathrm{v}_{0} \phi}$. Let $h$ be the label of this path and put $s_{\mathrm{v}}=s_{\mathrm{v}_{0}} h$. Since the length of $h$ is at most $m_{\mathrm{v}_{0} \phi}$, and since the action $h$ on $s_{\mathrm{v}_{0}}$ is defined, $s_{\mathrm{v}}$ belongs to $A^{*}$. By consistency of $\delta q_{\mathbf{D}}$, we have

$$
\mathrm{v} \delta q_{\mathbf{D}}=z_{\mathrm{v}_{0} \phi} s_{\mathrm{v}}
$$

Moreover, for every edge $\mathrm{e} \in \mathcal{E}_{\mathrm{v}, \omega}$,

$$
\mathrm{e} \delta q_{\mathbf{D}}=\mathrm{v} \delta q_{\mathbf{D}}=z_{\mathrm{v}_{0} \phi} s_{\mathrm{v}}
$$

Now, we deduce from Lemma 3.4 the existence of non-explicit operations $\pi_{v_{0} \phi}, \rho_{v}$ and $\rho_{\mathrm{e}}$ such that

$$
\begin{aligned}
\mathrm{v} \delta & =\rho_{\mathrm{v}} \cdot \pi_{\mathrm{v}_{0} \phi} \cdot s_{\mathrm{v}}, \\
\mathrm{e} \delta & =\rho_{\mathrm{e}} \cdot \pi_{\mathrm{v}_{0} \phi} \cdot s_{\mathrm{v}} \quad \text { for any edge } \mathrm{e} \in \mathcal{E}_{\mathrm{v}, \omega} .
\end{aligned}
$$

Suppose that the restriction of $\delta$ to $\mathcal{E}_{\mathrm{v}_{0} \phi}$ is not a commuting labelling. Then, by Lemma 2.5 of [9], $z_{\mathrm{v}_{0} \phi}=u^{-\infty} v$, for some explicit operations $u \neq 1$ and $v$, and, by Lemma 3.4, one can choose $\pi_{\mathrm{v}_{0} \phi}=u^{\omega} v$. In this case, we delete all the edges of $\mathcal{E}_{\mathrm{v}_{0} \phi}$ and let $\gamma_{1}$ and $\delta_{1}$ be the labellings of $\Gamma^{\prime}=\Gamma \backslash \mathcal{E}_{\mathrm{v}_{0} \phi}$ which differ from $\gamma$ and $\delta$ on the following edges and vertices in which they are defined by:

- $\mathrm{v} \gamma_{1}=\left(\rho_{\mathrm{v}} \cdot \pi_{\mathrm{v}_{0} \phi}\right) \psi$ and $\mathrm{v} \delta_{1}=\rho_{\mathrm{v}} \cdot \pi_{\mathrm{v}_{0} \phi}$, for each $\mathrm{v} \in \mathrm{v}_{0} \phi$;

- $\mathrm{e} \gamma_{1}=\left(\rho_{\mathrm{e}} \cdot \pi_{\mathrm{v}_{0} \phi}\right) \psi$ and $\mathrm{e} \delta_{1}=\rho_{\mathrm{e}} \cdot \pi_{\mathrm{v}_{0} \phi}$, for each edge e such that e $\alpha \notin \mathrm{v}_{0} \phi$ and $\mathrm{e} \omega \in \mathrm{v}_{0} \phi$

- $\mathrm{e} \gamma_{1}=\left(s_{\mathrm{e} \alpha} \cdot \rho_{\mathrm{e}} \cdot \pi_{\mathrm{v}_{0} \phi}\right) \psi$ and $\mathrm{e} \delta_{1}=s_{\mathrm{e} \alpha} \cdot \rho_{\mathrm{e}} \cdot \pi_{\mathrm{v}_{0} \phi}$, for each edge e such that e $\alpha \in \mathrm{v}_{0} \phi$ and $\mathrm{e} \omega \in \mathrm{v}_{0} \phi$;

- $\mathrm{e} \gamma_{1}=\left(s_{\mathrm{e} \alpha} \cdot \mathrm{e} \delta\right) \psi$ and $\mathrm{e} \delta_{1}=s_{\mathrm{e} \alpha} \cdot \mathrm{e} \delta$, for each edge e such that $\mathrm{e} \alpha \in \mathrm{v}_{0} \phi$ and $\mathrm{e} \omega \notin \mathrm{v}_{0} \phi$.

In these conditions, $\delta_{1} \psi=\gamma_{1}$ and, by Proposition 3.2, $\delta_{1} q_{\mathbf{L S I}}$ is consistent. Suppose that we construct a labelling $\delta_{1}^{\prime}$ of $\Gamma^{\prime}$ by rank $1 \kappa$-terms satisfying conditions (with a fixed integer $\left.M \geq m_{\mathrm{v}_{0} \phi}\right)$ : 
i) $\delta_{1}^{\prime} \psi=\gamma_{1}$ and $\delta_{1}^{\prime} p_{\mathbf{L S I}}$ is consistent;

ii) for any $\mathrm{g} \in \Gamma^{\prime}$, if $\mathrm{g} \delta_{1} q_{\mathbf{D}}=u^{-\infty} v$, where $u \neq 1$ and $v$ are finite words, then $\mathrm{g} \delta_{1}^{\prime} p_{\mathbf{D}}=$ $u^{-\infty} v$;

iii) for any $\mathrm{g} \in \Gamma^{\prime}$, if $\mathrm{g} \delta_{1}=u \sigma$, where $u \in A^{+}$and $\sigma \in \bar{\Omega}_{A} \mathbf{S}$ with $|u| \leq M$, then $\mathrm{g} \delta_{1}^{\prime}=u \sigma^{\prime}$ where $\sigma^{\prime} \in \Omega_{A}^{\kappa} \mathbf{S}$ is such that $\sigma \psi=\sigma^{\prime} \psi$. Hence, in particular, for each edge e such that e $\alpha \in \mathrm{v}_{0} \phi$, e $\delta_{1}^{\prime}=s_{\mathrm{e} \alpha} \cdot \sigma_{\mathrm{e}}$ for some $\sigma_{\mathrm{e}} \in \Omega_{A}^{\kappa} \mathbf{S}$;

iv) for any vertices $\mathbf{v}_{1}$ and $\mathbf{v}_{2}$, if $\mathbf{v}_{1} \delta_{1} q_{\mathbf{L S l}}=\mathbf{v}_{2} \delta_{1} q_{\mathbf{L S l}}$, then $\mathbf{v}_{1} \delta_{1}^{\prime} p_{\mathbf{L S l}}=\mathbf{v}_{2} \delta_{1}^{\prime} p_{\mathbf{L S l}}$. Notice that in this case, we have $\mathbf{v} \delta_{1}^{\prime} p_{\mathbf{L S l}}=\mathrm{v}_{0} \delta_{1}^{\prime} p_{\mathbf{L S l}}$, for each $\mathbf{v} \in \mathrm{v}_{0} \phi$.

Therefore, if we define $\delta^{\prime}$ to be the labelling of $\Gamma$ such that:

- $\delta^{\prime}$ coincides with $\delta_{1}^{\prime}$ on $\Gamma^{\prime}$;

- $\mathrm{v} \delta^{\prime}=\mathrm{v} \delta_{1}^{\prime} \cdot s_{\mathrm{v}}$, for each vertex $\mathrm{v} \in \mathrm{v}_{0} \phi$;

- $\mathrm{e} \delta^{\prime}=\mathrm{e} \delta_{1}^{\prime} \cdot s_{\mathrm{e} \omega}$, for each edge e such that e $\alpha \notin \mathrm{v}_{0} \phi$ and e $\omega \in \mathrm{v}_{0} \phi$;

- $\mathrm{e} \delta^{\prime}=\sigma_{\mathrm{e}} \cdot s_{\mathrm{e} \omega}$, for each edge e such that e $\alpha \in \mathrm{v}_{0} \phi$ and $\mathrm{e} \omega \in \mathrm{v}_{0} \phi$;

- $\delta^{\prime}=\sigma_{\mathrm{e}}$, for each edge e such that e $\alpha \in \mathrm{v}_{0} \phi$ and $\mathrm{e} \omega \notin \mathrm{v}_{0} \phi$;

- $\mathrm{e}^{\prime}=\mathrm{e} \delta$, for each edge $\mathrm{e} \in \mathcal{E}_{\mathrm{v}_{0} \phi}$;

then $\delta^{\prime}$ is a labelling of $\Gamma$ by $\Omega_{A}^{\kappa} \mathbf{S}$ such that $\delta^{\prime} \psi=\gamma$ and, by Proposition $5.1, \delta^{\prime} p_{\mathbf{L S I}}$ is consistent.

The case in which the restriction of $\delta$ to $\mathcal{E}_{\mathrm{v}_{0} \phi}$ is a commuting labelling can be treated analogously (in this case, condition ii) above is superfluous). Therefore, by induction on the number of $\phi$-classes $\mathrm{v}_{0} \phi$ such that $\mathcal{E}_{\mathrm{v}_{0} \phi}$ is non-empty, we may assume that all edges beginning in vertices labelled by non-explicit operations are labelled by non-explicit operations. Finally, we can easily eliminate edges labelled by explicit operations which begin in vertices labelled by explicit operations (and vertices labelled by explicit operations which are not the beginning of edges labelled by non-explicit operations). For that, it suffices to define the labels of these edges under $\delta^{\prime}$ to be equal to their labels under $\delta$ by the imposition of condition iii) above which preserves prefixes of any fixed length of labels of vertices. Therefore, we may assume that all edges are labelled by non-explicit operations.

Finally, we reduce to the case in which all vertices are labelled by non-explicit operations. Suppose that $v$ is a vertex labelled by an explicit operation and let $e_{1}, \ldots, e_{n}$ be all the edges beginning in $v$. Notice that, since all edges are labelled by non-explicit operations, $\mathrm{v}$ is not the end of an edge. Moreover, for each $i \in\{1, \ldots, n\}$, we can write

$$
\mathrm{e}_{i} \delta=\pi_{i} \rho_{i}
$$

for some non-explicit operations $\pi_{i}$ and $\rho_{i}$. Delete vertex $\vee$ and, for each $i \in\{1, \ldots, n\}$, delete edge $\mathrm{e}_{i}$, let $\mathrm{v}_{i}^{\prime}$ be a new vertex, let $\mathrm{e}_{i}^{\prime}$ be a new edge with beginning in $\mathrm{v}_{i}^{\prime}$ and end in $\mathrm{e}_{i} \omega$ and let $\gamma_{1}$ and $\delta_{1}$ be the labellings of $\Gamma^{\prime}=\left(\Gamma \backslash\left\{\mathrm{v}, \mathrm{e}_{1}, \ldots, \mathrm{e}_{n}\right\}\right) \cup\left\{\mathrm{v}_{i}^{\prime}, \mathrm{e}_{i}^{\prime} \mid i=1, \ldots, n\right\}$ defined as follows: 
- $\gamma_{1}$ and $\delta_{1}$ coincide, respectively, with $\gamma$ and $\delta$ on $\Gamma \backslash\left\{\mathrm{v}, \mathrm{e}_{1}, \ldots, \mathrm{e}_{n}\right\}$;

- $\mathrm{v}_{i}^{\prime} \gamma_{1}=\left(\mathrm{v} \delta \cdot \pi_{i}\right) \psi, \mathrm{v}_{i}^{\prime} \delta_{1}=\mathrm{v} \delta \cdot \pi_{i}, \mathrm{e}_{i}^{\prime} \gamma_{1}=\rho_{i} \psi$ and $\mathrm{e}_{i}^{\prime} \delta_{1}=\rho_{i}$, for each $i \in\{1, \ldots, n\}$.

Since $\delta \psi=\gamma$ and $\delta q_{\mathbf{L S I}}$ is consistent, it is clear that $\delta_{1} \psi=\gamma_{1}$ and $\delta_{1} q_{\mathbf{L S I}}$ is consistent. Suppose that there exists a labelling $\delta_{1}^{\prime}$ of $\Gamma^{\prime}$ by rank $1 \kappa$-terms satisfying conditions i) and iii) above (for condition iii) we have to fix $M \geq|\mathrm{v} \delta|$ so that, for each $i, \mathrm{v}_{i}^{\prime} \delta_{1}^{\prime}=\mathrm{v} \delta \cdot \sigma_{i}$ for some $\left.\sigma_{i} \in \Omega_{A}^{\kappa} \mathbf{S}\right)$. Then, let $\delta^{\prime}$ coincide with $\delta_{1}^{\prime}$ on $\Gamma \backslash\left\{\mathrm{v}, \mathrm{e}_{1}, \ldots, \mathrm{e}_{n}\right\}$, let $\mathrm{v} \delta^{\prime}=\mathrm{v} \delta$ and let $\mathrm{e}_{i} \delta^{\prime}=\sigma_{i} \cdot \mathrm{e}_{i}^{\prime} \delta_{1}^{\prime}$ for all $i$. Therefore $\delta^{\prime}$ is a labelling of $\Gamma$ by $\Omega_{A}^{\kappa} \mathbf{S}$ such that $\delta^{\prime} \psi=\gamma$ and $\delta^{\prime} p_{\mathbf{L S I}}$ is consistent. By induction on the number of vertices labelled by explicit operations under $\delta$, we may assume that all vertices are labelled by non-explicit operations.

We have proved the following result.

Proposition 6.1 A condition sufficient for the $\kappa$-reducibility of LSl is the existence, for a finite graph $\Gamma$ all of whose vertices and edges are labelled by non-explicit operations under $\delta$ and an integer $M$ large enough, of a labelling $\delta^{\prime}$ of $\Gamma$ satisfying conditions

c1) $\delta^{\prime} \psi=\gamma$ and $\delta^{\prime} p_{\mathbf{L S I}}$ is consistent;

c2) for any $\mathrm{g} \in \Gamma$, if $\mathrm{g} \delta q_{\mathbf{D}}=u^{-\infty} v$, where $u \neq 1$ and $v$ are finite words, then $\mathrm{g} \delta^{\prime} p_{\mathbf{D}}=$ $u^{-\infty} v$;

c3) for any $\mathrm{g} \in \Gamma$, if $\mathrm{g} \delta=u \sigma$, where $u \in A^{+}$and $\sigma \in \bar{\Omega}_{A} \mathbf{S}$ with $|u| \leq M$, then $\mathrm{g} \delta^{\prime}=u \sigma^{\prime}$ where $\sigma^{\prime} \in \Omega_{A}^{\kappa} \mathbf{S}$ is such that $\sigma \psi=\sigma^{\prime} \psi$;

c4) for any vertices $\mathbf{v}_{1}$ and $\mathrm{v}_{2}$, if $\mathbf{v}_{1} \delta q_{\mathbf{L S l}}=\mathrm{v}_{2} \delta q_{\mathbf{L S l}}$, then $\mathbf{v}_{1} \delta^{\prime} p_{\mathbf{L S l}}=\mathrm{v}_{2} \delta^{\prime} p_{\mathbf{L S l}}$.

We assume therefore that $\Gamma$ is a finite graph all of whose vertices and edges are labelled by non-explicit operations under $\delta$ and fix an integer $M$ large enough. The objective is to construct a labelling $\delta^{\prime}$ of $\Gamma$ in the conditions of Proposition 6.1.

\subsection{Reduction rule}

Before giving explicitly the algorithm, let us give some details of the process. For a sufficiently large integer $n$ (to be fixed below) and for each $\mathrm{g} \in \Gamma$ consider a word $u_{\mathrm{g} \delta} \in A^{+}$, given by Lemma 3.3. We will apply to each of these words a process of transformation described in sections 6.4 and 6.5 below. The $\kappa$-term obtained (in fact it is a rank $1 \kappa$-term), denoted $\widehat{u_{\mathrm{g} \delta}}$, will be chosen to be $\mathrm{g} \delta^{\prime}$. The process of transformation will use a unique reduction rule which consists in the substitution of certain factors by certain rank $1 \kappa$-terms. We proceed to describe (and to justify the use of) this rule.

Since $\psi$ is a homomorphism and so, in particular, commutes with $\omega$-powers, we deduce from Lemma 2.1 that for every word $a_{1} \cdots a_{k} \in A^{+}$of length $k$ (remember that $k=|S|$ by Definition 4.3),

$$
\left[a_{1} \cdots a_{k}\right] \psi=\left[a_{1} \cdots a_{i-1}\left(a_{i} \cdots a_{j}\right)^{\omega} a_{j+1} \cdots a_{k}\right] \psi
$$


for some integers $1 \leq i \leq j \leq k$. Therefore, for each word $x=a_{1} \cdots a_{k}$ of length $k$ we may fix a rank $1 \kappa$-term

$$
\bar{x}=a_{1} \cdots a_{i-1}\left(a_{i} \cdots a_{j}\right)^{\omega} a_{j+1} \cdots a_{k}
$$

such that $x \psi=\bar{x} \psi$.

Now, suppose that $x \in A^{+}$is a word such that $|x|>k$ and $S$ satisfies $x=x^{\omega}$. Let $y \in A^{+}$be the unique primitive word such that $x$ is a power of $y$. In this case we define

$$
\bar{x}=y^{\omega}
$$

and, so, also in this case the equality $x \psi=\bar{x} \psi$ holds.

Let $\bar{A}$ be the alphabet

$$
\bar{A}=A \cup\left\{\bar{x} \mid x \in A^{+} \text {and } \bar{x} \text { is defined }\right\} .
$$

Definition 6.2 (reduction rule) The reduction rule (to be applied on terms in the alphabet $\bar{A}$ ) is the following:

R) $t_{1} x t_{2} \rightarrow t_{1} \bar{x} t_{2}$, where $t_{1}, t_{2} \in \bar{A}^{*}, x \in A^{+}$and $\bar{x}$ is defined.

Notice that:

- rule $R$ ) substitutes occurrences of certain factors $x \in A^{+}$, with $|x| \geq k$, by elements $\bar{x}$ of the alphabet $\bar{A}$; therefore, this is clearly a Nœtherian system since rule $R$ ) reduces the length of terms in the alphabet $\bar{A}$;

- rule $R$ ) applies explicit operations and rank $1 \kappa$-terms to rank $1 \kappa$-terms;

- $\left(t_{1} x t_{2}\right) \psi=\left(t_{1} \bar{x} t_{2}\right) \psi$; since Lemma 3.3 states that $S$ satisfies $\mathrm{g} \delta=u_{\mathrm{g} \delta}$, this equality will guarantee that $\delta^{\prime} \psi=\gamma$.

Notice that, since we want to guarantee the consistency of $\delta^{\prime} p_{\mathbf{L S l}}$, the pseudoidentity $\widehat{u_{\mathrm{e} \alpha \delta}} \cdot \widehat{u_{\mathrm{e} \delta}}=\widehat{u_{\mathrm{e} \omega \delta}}$ must hold in LSl for every edge e of $\Gamma$. Hence, if $\widehat{u_{\mathrm{e} \alpha \delta}} \cdot \widehat{u_{\mathrm{e} \delta}}$ has a factor of the form $\bar{x} y \bar{z}$ with $x, z \in A^{+}$and $y \in A^{*}$, then $\bar{x} y \bar{z}=x_{1} x_{2}^{\omega} x_{3} y z_{1} z_{2}^{\omega} z_{3}$ for some $x_{1}, x_{3}, y, z_{1}, z_{3} \in A^{*}$ and $x_{2}, z_{2} \in A^{+}$, and, by Proposition 5.1, $x_{2}^{\omega} x_{3} y z_{1} z_{2}^{\omega}$ must also occur in $\widehat{u_{\mathrm{e} \omega} \delta}$. For that, it suffices to guarantee that $\bar{x} y \bar{z}$ is also a factor of $\widehat{u_{\mathrm{e}} \omega \delta}$. Reciprocally, we will guarantee that every factor of $\widehat{u_{\mathrm{e} \omega} \delta}$ of the form $\bar{x} y \bar{z}$, with $x, z \in A^{+}$ and $y \in A^{*}$, is also a factor of $\widehat{u_{\mathrm{e} \alpha} \delta} \cdot \widehat{u_{\mathrm{e} \delta}}$. Moreover, the substitutions will be made in such a way that the unique prefix (resp. suffix) of $\widehat{u_{\mathrm{e} \alpha \delta}} \cdot \widehat{u_{\mathrm{e} \delta}}$ of the form $y \bar{z}$ (resp. $\bar{z} y$ ) with $y \in A^{*}$ and $z \in A^{+}$is also a prefix (resp. suffix) of $\widehat{u_{\mathrm{e} \omega \delta}}$.

\subsection{Factorizations of the words $u_{\mathrm{g} \delta}$}

The objective of this subsection is, for each $\mathrm{g} \in \Gamma$, to identify in the word $u_{\mathrm{g} \delta}$ the first and the last occurrences of factors on which rule $R$ ) is going to be applied. These applications of rule $R$ ) will determine the unique prefix (resp. suffix) of $\widehat{u_{\mathrm{g} \delta}}$ of the form $y \bar{z}$ (resp. $\bar{z} y$ ) with $y \in A^{*}$ and $z \in A^{+}$. 
As noticed in the end of last section, we want to guarantee in particular that, for every edge e of $\Gamma, \widehat{u_{\mathrm{e} \alpha \delta}} \cdot \widehat{u_{\mathrm{e} \delta}}$ and $\widehat{u_{\mathrm{e} \omega} \delta}$ have the same factors of the form $\bar{x} y \bar{z}$ with $x, z \in A^{+}$ and $y \in A^{*}$. Since the last occurrence of a factor of the form $\bar{x}$ in $\widehat{u_{\mathrm{e} \alpha \delta}}$ and the first occurrence of a factor of the form $\bar{z}$ in $\widehat{u_{\mathrm{e} \delta}}$ originate a factor of the form $\bar{x} y \bar{z}$ in $\widehat{u_{\mathrm{e} \alpha} \delta} \cdot \widehat{u_{\mathrm{e} \delta}}$, we must guarantee that $\bar{x} y \bar{z}$ is also a factor of $\widehat{u_{\mathrm{e \omega}} \delta}$ (the word $x y z \in A^{+}$will be called the center of e). On the other hand, since $\mathbf{L S l}$ verifies the pseudoidentity $a^{\omega} b a^{\omega} b a^{\omega}=a^{\omega} b a^{\omega}$, it may happen for instance that the word $x y z$ has only one occurrence in $u_{\mathrm{e} \alpha \delta} \cdot u_{\mathrm{e} \delta}$ and "many" occurrences in $u_{\mathrm{e} w \delta}$ or vice-versa. This may constitute a problem since we want that $\widehat{u_{\mathrm{e} \alpha} \delta} \cdot \widehat{u_{\mathrm{e} \delta}}$ and $\widehat{u_{\mathrm{e} \omega} \delta}$ have the same factors of the form $\bar{x} y \bar{z}$. Therefore, we must localize all occurrences of the word $x y z$ in $u_{\mathrm{e} \alpha \delta} \cdot u_{\mathrm{e} \delta}$ and $u_{\mathrm{e} \omega \delta}$ and substitute $x$ by $\bar{x}$ and $z$ by $\bar{z}$. In fact, we may not be able to substitute all these occurrences since they may be not disjoint and rule $R$ ) can only be applied on occurrences of factors of $A^{+}$which are disjoint. So these factors $x y z \in A^{+}$will be chosen to permit substitutions on certain occurrences which are conveniently "separated" by a process, that uses the results of Section 4.1, that we proceed to describe.

Let $\mathrm{g} \in \Gamma$. We denote by $\mathrm{g} \theta$ the connected component of $\mathrm{g}$ in the graph $\Gamma$, by $\mathcal{V}_{\mathrm{g} \theta}$ the set of vertices of $\mathrm{g} \theta$ and by $\mathcal{E}_{\mathrm{g} \theta}$ the set of edges of $\mathrm{g} \theta$. We denote also

$$
\mathbf{k}_{\mathrm{g}}=\mathrm{g} \delta q_{\mathbf{K}} \quad \text { and } \quad \mathbf{d}_{\mathrm{g}}=\mathrm{g} \delta q_{\mathbf{D}} .
$$

Notice that $\mathbf{k}_{\mathrm{g}} \in A^{\mathbb{N}}$, that $\mathbf{d}_{\mathrm{g}} \in A^{-\mathbb{N}}$ and, by consistency of $\delta q_{\mathbf{D}}$, that $\mathbf{d}_{\mathrm{e}}=\mathbf{d}_{\mathrm{e} \omega}$ for any edge e. Moreover, the consistency of $\delta q_{\mathbf{K}}$ implies the equality $\mathbf{k}_{\mathrm{v}}=\mathbf{k}_{\mathrm{v}^{\prime}}$, for all vertices $\mathbf{v}$ and $\mathrm{v}^{\prime}$ in the same connected component. We then denote, for any vertex $\mathrm{v}, \mathbf{k}_{\mathrm{v} \theta}=\mathbf{k}_{\mathrm{v}}$.

Let $M$ be the integer fixed after Proposition 6.1. For each vertex $\vee$ and for each edge e, let

$$
\begin{aligned}
& l_{\mathrm{v}}=\mathbf{k}_{\mathrm{v} \theta}[1, M+k], \\
& l_{\mathrm{e}}=\mathbf{k}_{\mathrm{e}}\left[1, i_{\mathrm{e}}\right], \quad \text { where } i_{\mathrm{e}} \geq M+k \text { is an integer defined below, } \\
& r_{\mathrm{v}}=\mathbf{d}_{\mathrm{v}}\left[-i_{\mathrm{v}},-1\right], \quad \text { where } i_{\mathrm{v}} \geq M+k \text { is an integer defined below, } \\
& r_{\mathrm{e}}=r_{\mathrm{e} \omega} .
\end{aligned}
$$

Definition 6.3 (words $\mathrm{w}_{\mathrm{e}}$ and $c_{\mathrm{e}}$ ) For each edge $\mathrm{e}$, let $\mathrm{w}_{\mathrm{e}}$ be the bi-infinite word

$$
\mathbf{w}_{\mathrm{e}}=\mathbf{d}_{\mathrm{e} \alpha} \cdot \mathbf{k}_{\mathrm{e}}
$$

and let $c_{\mathrm{e}} \in A^{+}$, called the center of $\mathrm{e}$, be the following center of $\mathbf{w}_{\mathrm{e}}$

$$
c_{\mathrm{e}}=r_{\mathrm{e} \alpha} l_{\mathrm{e}}=\mathbf{w}_{\mathrm{e}}\left[-i_{\mathrm{e} \alpha}, i_{\mathrm{e}}[.\right.
$$

We say also that $c_{\mathrm{e}}$ is a center of $\Gamma$. The center $c_{\mathrm{e}}$ is said to be periodic if and only if the bi-infinite word $\mathbf{w}_{\mathrm{e}}$ is periodic.

Definition 6.4 (integers $i_{\mathrm{e}}$ and $i_{\mathrm{v}}$ ) For $\mathrm{e} \in \mathcal{E}(\Gamma)$ and $\vee \in \mathcal{V}(\Gamma), i_{\mathrm{e}}$ and $i_{\mathrm{v}}$ in (9) and (10), respectively, are integers chosen sufficiently large so that:

(a) if $\mathrm{e}^{\prime}$ is an edge such that $\mathbf{w}_{\mathrm{e}} \sim \mathbf{w}_{\mathrm{e}^{\prime}}$, then $c_{\mathrm{e}}=c_{\mathrm{e}^{\prime}}$ (not necessarily $r_{\mathrm{e} \alpha}=r_{\mathrm{e}^{\prime} \alpha}$ and $\left.l_{\mathrm{e}}=l_{\mathrm{e}^{\prime}}\right)$. 
(b) If $\mathbf{k}_{\mathrm{e}}$ is ultimately periodic, say $\mathbf{k}_{\mathrm{e}}=v u^{+\infty}$ where $u$ is the unique period of $\mathbf{k}_{\mathrm{e}}$ which is a Lyndon word, then $l_{\mathrm{e}}=v u^{p}$ for some positive integer $p$ such that $S$ satisfies $u^{\omega}=u^{p}$.

(c) If $\mathbf{d}_{\sqrt{ }}$ is ultimately periodic, say $\mathbf{d}_{\mathrm{v}}=u^{-\infty} v$ where $u$ is the unique period of $\mathbf{d}_{\sqrt{ }}$ which is a Lyndon word, then $r_{\mathrm{v}}=u^{p} v$ for some positive integer $p$ such that $S$ satisfies $u^{\omega}=u^{p}$.

(d) For every $\mathrm{g} \in \Gamma$, if $\mathbf{w}_{\mathrm{e}}$ is not a bi-infinite factor of $\mathrm{g} \delta$, then $c_{\mathrm{e}}$ is not a factor of $\mathrm{g} \delta$.

(e) $i_{\mathrm{e}} \geq Q$, where, for $B=\left\{\mathbf{w}_{\mathrm{e}} \mid \mathrm{e} \in \mathcal{E}(\Gamma)\right\}, Q$ is a positive integer in the conditions of Lemma 4.2.

Notice that $i_{\mathrm{e}}$ and $i_{\mathrm{e} \omega}$ may effectively be chosen satisfying the conditions above since, for instance, if e and $\mathrm{e}^{\prime}$ are edges such that $\mathbf{w}_{\mathrm{e}} \sim \mathbf{w}_{\mathrm{e}^{\prime}}$ and $\mathbf{k}_{\mathrm{e}}=v u^{+\infty}$ is ultimately periodic, then $\mathbf{k}_{\mathrm{e}^{\prime}}$ is also ultimately periodic of the form $\mathbf{k}_{\mathrm{e}^{\prime}}=v^{\prime} u^{+\infty}$ and one of $v$ or $v^{\prime}$ is a suffix of the other.

Notice also that, if $\mathrm{e}$ is an edge such that $\mathbf{w}_{\mathrm{e}}$ is ultimately periodic, say of the form $u^{-\infty} v x^{+\infty}$ where $u$ and $x$ are Lyndon words, then $c_{\mathrm{e}}=u^{p} v x^{q}$ for some positive integers $p$ and $q$ such that $S$ satisfies $u^{\omega}=u^{p}$ and $x^{\omega}=x^{q}$. In particular: if $\mathbf{w}_{\mathrm{e}}$ is periodic, then $u=x$ and $v$ is a power of $u$, so that we can write $c_{\mathrm{e}}=u^{h} u^{i} u^{h}=u^{2 h+i}$ for some integers $h>0$ and $i \geq 0$ such that $S$ satisfies $u^{\omega}=u^{h}$ [one can choose, for instance $h=\min (p, q)]$; if $\mathbf{w}_{\mathrm{e}}$ is non-periodic, then $c_{\mathrm{e}}=u^{p} v x^{q}$ is not a (non-trivial) power of a word.

Since, for each edge e, the word $c_{\mathrm{e}}$ is a center of the bi-infinite word $\mathbf{w}_{\mathrm{e}}$ and $i_{\mathrm{e}} \geq Q$, Lemma 4.2 guarantees the existence of an integer $T \geq \max \left\{i_{\mathrm{v}} \mid \mathrm{v} \in \mathcal{V}(\Gamma)\right\}$ such that, for every edges e and $\mathrm{e}^{\prime}$, with $\mathbf{w}_{\mathrm{e}}$ and $\mathbf{w}_{\mathrm{e}^{\prime}}$ non-periodic and $\mathbf{w}_{\mathrm{e}} \not \mathbf{w}_{\mathrm{e}^{\prime}}, \overleftarrow{c_{\mathrm{e}}}=\mathbf{w}_{\mathrm{e}}\left[-T, i_{\mathrm{e}}[\right.$ and $\overleftarrow{c_{\mathrm{e}^{\prime}}}=\mathbf{w}_{\mathrm{e}^{\prime}}\left[-T, i_{\mathrm{e}^{\prime}}\left[\right.\right.$, and every word $u \in A^{+}$

- if two occurrences of $c_{\mathrm{e}}$ and $c_{\mathrm{e}^{\prime}}$ are allowed in $u$ relatively to $\overleftarrow{c_{\mathrm{e}}}$ and $\overleftarrow{c_{\mathrm{e}^{\prime}}}$ respectively then these occurrences of $c_{\mathrm{e}}$ and $c_{\mathrm{e}^{\prime}}$ are disjoint;

- any two distinct allowed occurrences of $c_{\mathrm{e}}$ in $u$, relatively to $\overleftarrow{c_{\mathrm{e}}}$, are disjoint.

For each edge e such that $\mathbf{w}_{\mathrm{e}}$ is non-periodic, the left-extension $\overleftarrow{c_{\mathrm{e}}}=\mathbf{w}_{\mathrm{e}}\left[-T, i_{\mathrm{e}}[\right.$ of the center $c_{\mathrm{e}}$ is called the extended-center of e.

Finally, we fix the integer $n$, mentioned in the beginning of Section 6.2.

Definition 6.5 (constants $L$ and $n$, and words $u_{\mathrm{g} \delta}$ ) Let $L$ be the maximal length of the centers and extended-centers of $\Gamma$. That is

$$
L=\max \left\{\left|\overleftarrow{c_{\mathrm{e}}}\right|,\left|c_{\mathrm{f}}\right| \mid \mathrm{e}, \mathrm{f} \in \mathcal{E}(\Gamma) \text { with } c_{\mathrm{f}} \text { periodic and } c_{\mathrm{e}} \text { non-periodic }\right\}
$$

Then we fix an integer $n>3 L+k^{\prime \prime}$ and, for each $\mathrm{g} \in \Gamma$, we consider a word $u_{\mathrm{g} \delta} \in A^{+}$, given by Lemma 3.3. 
Let $\mathrm{g} \in \Gamma$. We want to localize in $u_{\mathrm{g} \delta}$ the allowed occurrences of the non-periodic centers of $\Gamma$. Since these occurrences are allowed in $u_{\mathrm{g} \delta}$ if and only if they occur as suffixes of the respective extended-centers, the occurrences that occur "near" the beginning of $u_{\mathrm{g} \delta}$ are not allowed. If $\mathrm{g}$ is a vertex, this does not constitute a problem, but if $\mathrm{g}$ is an edge e this is not desirable since we want to guarantee that LSI satisfies $\widehat{u_{\mathrm{e} \alpha \delta}} \cdot \widehat{u_{\mathrm{e} \delta}}=\widehat{u_{\mathrm{e} \omega} \delta}$. We overcome this situation by localizing in $u_{\mathrm{e} \alpha \delta} \cdot u_{\mathrm{e} \delta}$ the allowed occurrences of the non-periodic centers of $\Gamma$. Then, an occurrence of a center $c_{\mathrm{f}}$ in $u_{\mathrm{e} \alpha \delta} \cdot u_{\mathrm{e} \delta}$ which occurs in $u_{\mathrm{e} \delta}$ will be said to be allowed in $u_{\mathrm{e} \delta}$ if it is an allowed occurrence in $u_{\mathrm{e} \alpha \delta} \cdot u_{\mathrm{e} \delta}$.

Notice that $l_{\mathrm{g}}$ is a prefix of $u_{\mathrm{g} \delta}$ and $r_{\mathrm{g}}$ is a suffix of $u_{\mathrm{g} \delta}$, so that we can write

$$
u_{\mathrm{g} \delta}=l_{\mathrm{g}} u_{\mathrm{g}} r_{\mathrm{g}}
$$

for some $u_{\mathrm{g}} \in A^{+}$. Now, we localize in $u_{\mathrm{g} \delta}$ every allowed occurrences of the nonperiodic centers $c_{\mathrm{e}}$ of $\Gamma$. As observed above, the allowed occurrences of non-periodic centers are disjoint. Moreover, assuming that $T$ was chosen large enough, these occurrences are also disjoint from the prefix $l_{\mathrm{g}}$ of $u_{\mathrm{g} \delta}$. This is clear when $\mathrm{g}$ is a vertex. When $\mathrm{g}$ is an edge, then an occurrence of a center $c_{\mathrm{e}}$ in $u_{\mathrm{g} \delta}$ is allowed if the corresponding occurrence is allowed in $u_{\mathrm{g} \alpha \delta} \cdot u_{\mathrm{g} \delta}$. Now, as

$$
u_{\mathrm{g} \alpha \delta} \cdot u_{\mathrm{g} \delta}=l_{\mathrm{g} \alpha} u_{\mathrm{g} \alpha} r_{\mathrm{g} \alpha} \cdot l_{\mathrm{g}} u_{\mathrm{g}} r_{\mathrm{g}}=l_{\mathrm{g} \alpha} u_{\mathrm{g} \alpha} c_{\mathrm{g}} u_{\mathrm{g}} r_{\mathrm{g}}
$$

and the occurrence of $c_{\mathrm{g}}$ signed in this factorization is allowed (since $u_{\mathrm{g} \alpha \delta}$ has the same suffix of $g \alpha \delta$ of length $n>T$ ), the occurrence of $c_{\mathrm{e}}$ in $u_{\mathrm{g} \delta}$ occurs in $u_{\mathrm{g}} r_{\mathrm{g}}$ and so it is disjoint from $l_{\mathrm{g}}$. Analogously, we may admit that the allowed occurrences of centers are also disjoint from the suffix $r_{\mathrm{g}}$ of $u_{\mathrm{g} \delta}$. Indeed, consider an allowed occurrence of a center $c_{\mathrm{e}}$ in $u_{\mathrm{g} \delta}$ and consider the word $u_{\mathrm{g} \delta} \cdot l_{\mathrm{f}}$ where $\mathrm{f}$ is an edge beginning in $\mathrm{g}$ or in $\mathrm{g} \omega$ whether $\mathrm{g}$ is a vertex or not (we assume that every vertex of $\Gamma$ is the beginning of some edge, by adding new vertices and edges if necessary). Then the corresponding occurrence of $c_{\mathrm{e}}$ in $u_{\mathrm{g} \delta} \cdot l_{\mathrm{f}}$ is allowed. Since $u_{\mathrm{g} \delta} \cdot l_{\mathrm{f}}=l_{\mathrm{g}} u_{\mathrm{g}} r_{\mathrm{g}} \cdot l_{\mathrm{f}}=l_{\mathrm{g}} u_{\mathrm{g}} c_{\mathrm{f}}$ and the occurrence of the suffix $c_{\mathrm{f}}$ is allowed in $u_{\mathrm{g} \delta} \cdot l_{\mathrm{f}}$, we deduce that the occurrence of $c_{\mathrm{e}}$ in $u_{\mathrm{g} \delta}$ occurs in $l_{\mathrm{g}} u_{\mathrm{g}}$ and so it is disjoint from $r_{\mathrm{g}}$. We notice that these last comments show that, when $\mathrm{g}$ is a vertex, the allowed occurrences of centers in $u_{\mathrm{g} \delta}$ are the same as the allowed occurrences of centers in $u_{\mathrm{g} \delta} \cdot u_{\mathrm{f} \delta}$ which occur in $u_{\mathrm{g} \delta}$, where $\mathrm{f}$ is an edge beginning in $\mathrm{g}$. Therefore, the process of allowing occurrences of centers used for edges is not necessary for vertices.

Now, if $c_{\mathrm{e}}=x^{i}$ is a periodic center of $\Gamma$ that occurs in $u_{\mathrm{g} \delta}$, then, by condition $(d)$ above, $x^{\omega}$ is a bi-infinite factor of $\mathrm{g} \delta$. Therefore, since $n>3 L$ and $\mathrm{g} \delta$ and $u_{\mathrm{g} \delta}$ have the same factors of length $n, x^{j}$ is a factor of $u_{\mathrm{g}}$ for a sufficiently large integer $j$ to guarantee that we can choose one occurrence of $c_{\mathrm{e}}$ disjoint from the allowed occurrences of the other centers and from $l_{\mathrm{g}}$ and $r_{\mathrm{g}}$.

The process above permits to obtain a factorization of $u_{\mathrm{g} \delta}$ as described in the following lemma.

Lemma 6.6 There is a factorization

$$
u_{\mathrm{g} \delta}=l_{\mathrm{g}} u_{\mathrm{g}, 0} c_{\mathrm{g}, 1} u_{\mathrm{g}, 1} c_{\mathrm{g}, 2} \cdots c_{\mathrm{g}, s_{\mathrm{g}}} u_{\mathrm{g}, s_{\mathrm{g}}} r_{\mathrm{g}}
$$

of $u_{\mathrm{g} \delta}$ such that 
- $s_{\mathrm{g}} \geq 0$

- $c_{\mathrm{g}, 1}, \ldots, c_{\mathrm{g}, s_{\mathrm{g}}}$ are centers of $\Gamma$, called the allowed centers of $u_{\mathrm{g} \delta}$;

- $u_{\mathrm{g}, 0}, u_{\mathrm{g}, 1}, \ldots, u_{\mathrm{g}, s_{\mathrm{g}}} \in A^{*}$;

- if $c_{\mathrm{g}, i}$ is a non-periodic center, then the factorization contains every allowed occurrence of $c_{\mathrm{g}, i}$;

- if $c_{\mathrm{g}, i}$ is a periodic center and $c_{\mathrm{g}, i}$ is a factor of $u_{\mathrm{g} \delta}$, then the factorization contains one occurrence of $c_{\mathrm{g}, i}$.

Definition 6.7 (factorization of $u_{\mathrm{g} \delta}$ for the centers of $\Gamma$ ) We fix a factorization of $u_{\mathrm{g} \delta}$ as in Lemma 6.6 and we say that this is the factorization of $u_{\mathrm{g} \delta}$ for the centers of $\Gamma$.

\subsection{Transformations on the words $l_{\mathrm{g}}, r_{\mathrm{g}}$ and $c_{\mathrm{g}}$}

Let $\mathrm{v}$ be a vertex. By $(8), l_{\mathrm{v}}=\mathbf{k}_{\mathrm{v} \theta}[1, M+k]$. Using the notation introduced in Section 6.2 , let

$$
\widehat{l_{\mathrm{v}}}=\mathbf{k}_{\mathrm{v} \theta}[1, M] \cdot \overline{\left.\left.\mathbf{k}_{\mathrm{v} \theta}\right] M, M+k\right]} .
$$

Moreover, by (10), $r_{\mathrm{v}}=\mathbf{d}_{\mathrm{v}}\left[-i_{\mathrm{v}},-1\right]$. If $\mathbf{d}_{\mathrm{v}}$ is ultimately periodic, say $\mathbf{d}_{\mathrm{v}}=u^{-\infty} v$ where $u$ is a Lyndon word, then, by condition (c) on Definition $6.4, r_{\mathrm{v}}=u^{p} v$ where $p$ is a positive integer such that $S$ satisfies $u^{\omega}=u^{p}$. In this case, let

$$
\widehat{r_{\mathrm{v}}}=\overline{u^{p}} \cdot v=u^{\omega} v .
$$

If $\mathbf{d}_{\vee}$ is not ultimately periodic, let

$$
\widehat{r_{\mathrm{v}}}=\overline{\mathbf{d}_{\mathrm{v}}\left[-i_{\mathrm{v}},-i_{\mathrm{v}}+k[\right.} \cdot \mathbf{d}_{\mathrm{v}}\left[-i_{\mathrm{v}}+k,-1\right] .
$$

Consider now an edge e. By (11), $r_{\mathrm{e}}=r_{\mathrm{e} \omega}$. We then let

$$
\widehat{r_{\mathrm{e}}}=\widehat{\mathrm{e}_{\mathrm{e}}} \text {. }
$$

On the other hand, by $(9), l_{\mathrm{e}}=\mathbf{k}_{\mathrm{e}}\left[1, i_{\mathrm{e}}\right]$. If $\mathbf{k}_{\mathrm{e}}$ is ultimately periodic, say $\mathbf{k}_{\mathrm{e}}=v u^{+\infty}$ where $u$ is a Lyndon word, then, by condition (b) on Definition $6.4, l_{\mathrm{e}}=v u^{p}$ where $p$ is a positive integer such that $S$ satisfies $u^{\omega}=u^{p}$. In this case, let

$$
\widehat{l_{\mathrm{e}}}=v \cdot \overline{u^{p}}=v u^{\omega} .
$$

If $\mathbf{k}_{\mathrm{e}}$ is not ultimately periodic, let

$$
\widehat{l_{\mathrm{e}}}=\mathbf{k}_{\mathrm{e}}\left[1, i_{\mathrm{e}}-k\right] \cdot \overline{\left.\left.\mathbf{k}_{\mathrm{e}}\right] i_{\mathrm{e}}-k, i_{\mathrm{e}}\right]} .
$$

Remember that $c_{\mathrm{e}}=r_{\mathrm{e} \alpha} l_{\mathrm{e}}$. Hence, we define at last

$$
\widehat{c_{\mathrm{e}}}=\widehat{r_{\mathrm{e} \alpha}} \cdot \widehat{l_{\mathrm{e}}} \text {. }
$$

Notice that $\widehat{c_{\mathrm{e}}}$ is a rank $1 \kappa$-term of the form $\bar{x} y \bar{z}=x_{1} x_{2}^{\omega} x_{3} y z_{1} z_{2}^{\omega} z_{3}$, with $x_{1}, x_{3}, y, z_{1}, z_{3} \in$ $A^{*}$ and $x_{2}, z_{2} \in A^{+}$. Moreover, if the bi-infinite word $\mathbf{w}_{\mathrm{e}}$ is ultimately periodic, then $x_{1}=x_{3}=z_{1}=z_{3}=1$ and $x_{2}{ }^{-\infty} y z_{2}{ }^{+\infty}$ is the $\sim$-class of $\mathbf{w}_{\mathrm{e}}$. 


\subsection{Transformations on the words $u_{\mathrm{g}, i}$}

In this section, we describe a process to make correspond a $\kappa$-term $\widehat{u_{\mathrm{g}, i}}$ to each word $u_{\mathrm{g}, i}$ $\left(i=0, \ldots, s_{\mathrm{g}}\right)$ in the factorization of $u_{\mathrm{g} \delta}$ for the centers of $\Gamma$ (see Definition 6.7). The process of transformation is the following:

First step Consider the marked factorization, described in Lemma 4.4,

$$
u_{\mathrm{g}, i}=x_{0} w_{1} x_{1} w_{2} \cdots w_{r} x_{r}
$$

of $u_{\mathrm{g}, i}$. If $\left|u_{\mathrm{g}, i}\right|<3 k-1$, then $u_{\mathrm{g}, i}=w_{1}$. In this case, let $\widehat{w_{1}}=w_{1}$. Suppose now that $\left|u_{\mathrm{g}, i}\right| \geq 3 k-1$. Then, by definition of marked factors, it is clear that $\left|w_{j}\right| \geq 2 k$ for every $j \in\{1, \ldots, r\}$, so that we can write $w_{j}=w_{j, 1} w_{j, 2} w_{j, 3}$ for some words $w_{j, 1}, w_{j, 2}$ and $w_{j, 3}$ with $\left|w_{j, 1}\right|=\left|w_{j, 3}\right|=k$. We define $\widehat{w_{j}}=\overline{w_{j, 1}} w_{j, 2} \overline{w_{j, 3}}$.

We let $u_{\mathrm{g}, i}(1)$ be the following rank $1 \kappa$-term

$$
u_{\mathrm{g}, i}(1)=x_{0} \widehat{w_{1}} x_{1} \widehat{w_{2}} x_{2} \cdots x_{r-1} \widehat{w_{r}} x_{r}
$$

Second step Define

$$
\widehat{x_{0}}= \begin{cases}x_{0} & \text { if }\left|x_{0}\right|<k \\ \overline{x_{0,1}} x_{0,2} & \text { if }\left|x_{0}\right| \geq k\end{cases}
$$

where $x_{0,1}$ is the prefix of length $k$ of $x_{0}$ and $x_{0}=x_{0,1} x_{0,2}$. Symmetrically, let

$$
\widehat{x_{r}}= \begin{cases}x_{r} & \text { if }\left|x_{r}\right|<k \\ x_{r, 1} \overline{x_{r, 2}} & \text { if }\left|x_{r}\right| \geq k\end{cases}
$$

where $x_{r, 2}$ is the suffix of length $k$ of $x_{r}$ and $x_{r}=x_{r, 1} x_{r, 2}$. Now, let

$$
u_{\mathrm{g}, i}(2)=\widehat{x_{0}} \widehat{w_{1}} x_{1} \widehat{w_{2}} x_{2} \cdots x_{r-1} \widehat{w_{r}} \widehat{x_{r}}
$$

Third step Let $y \in A^{+}$be a factor of $u_{\mathrm{g}, i}$ such that $2 k \leq|y|<3 k$. Two cases may arise.

First case Every extension of length $3 k-1$ in $u_{\mathrm{g}, i}$, of an occurrence of $y$, is a marked occurrence (see Section 4.2). In this case every occurrence of $y$ in $u_{\mathrm{g}, i}$ is contained in the marked factors.

Second case There exists a free occurrence in $u_{\mathrm{g}, i}$ of an extension $\tilde{y}$, of length $3 k-1$, of an occurrence of $y$. In this case, by definition of free occurrence (cf. Section 4.2), there exists a $k^{\prime \prime}$-neighborhood $v$ of $\tilde{y}$ such that $v$ is $k^{\prime}$-abundant. In particular, $\operatorname{docc}(\tilde{y}, v) \geq k^{\prime}$. Furthermore, every occurrence of a factor $z$ of length $3 k-1$ in the $k^{\prime \prime}$-neighborhood $v$ is free. This means that at least $k^{\prime}-2$ of the disjoint occurrences of $\tilde{y}$ in $v$ occur disjoint of the marked factors. More precisely, there exists an integer $0 \leq j \leq r \operatorname{such}$ that $\operatorname{docc}\left(\tilde{y}, x_{j}\right) \geq k^{\prime}-2$. Hence, since $y$ is a factor of $\tilde{y}, \operatorname{docc}\left(y, x_{j}\right) \geq k^{\prime}-2$. In this case, we say that $y$ has a free occurrence in $u_{\mathrm{g}, i}$. 
Consider the set $F$ of all factors $y$ of $u_{\mathrm{g}, i}$ such that $2 k \leq|y|<3 k$ and $y$ has a free occurrence in $u_{\mathrm{g}, i}$. By the second case above, for each $y \in F$ there exists an integer $0 \leq j \leq r$ such that $\operatorname{docc}\left(y, x_{j}\right) \geq k^{\prime}-2$. Therefore, the choice of $k^{\prime}$ allows us to select an occurrence for each $y \in F$ in such a way that these occurrences are pairwise disjoint. These occurrences are selected in the factors $x_{j}$ and, if $j=0$ or $j=r$, then we can select them, respectively, in $x_{0,2}$ and in $x_{r, 1}$. Since $2 k \leq|y|<3 k$, we can write

$$
y=y_{1} y_{2} y_{3}
$$

for some words $y_{1}, y_{2}$ and $y_{3}$ with $\left|y_{1}\right|=\left|y_{3}\right|=k$. We substitute in $u_{\mathrm{g}, i}(2)$ the selected occurrence of $y$ by $\widehat{y}=\overline{y_{1}} y_{2} \overline{y_{3}}$. We then obtain a term $u_{\mathrm{g}, i}(3)$.

Fourth step In this step, we admit the substitution of any occurrence, in the factors of $u_{\mathrm{g}, i}(3)$ which were obtained from the transformations on the words $x_{j}$, of a factor $y \in A^{+}$of length $k$ by $\bar{y}$. We say that a term obtained from $u_{\mathrm{g}, i}(3)$ using these substitutions is irreducible when it is not possible to make more substitutions (that is, when there are no more occurrences of factors $y \in A^{+}$of length $k$ on the factors which resulted from substitutions on the words $x_{j}$ ). We choose an irreducible term and denote it by $u_{\mathrm{g}, i}(4)$. This concludes the process of transformation of the word $u_{\mathrm{g}, i}$.

We let $\widehat{u_{\mathrm{g}, i}}=u_{\mathrm{g}, i}(4)$. This concludes the process of transformation of the factors of $u_{\mathrm{g} \delta}$ in its factorization for the centers of $\Gamma$. We then let

$$
\widehat{u_{\mathrm{g} \delta}}=\widehat{l_{\mathrm{g}}} \widehat{u_{\mathrm{g}, 0}} \widehat{c_{\mathrm{g}, 1}} \cdots \widehat{c_{\mathrm{g}, s_{\mathrm{g}}}} \widehat{u_{\mathrm{g}, s_{\mathrm{g}}}} \widehat{r_{\mathrm{g}}} .
$$

Definition 6.8 (labelling $\delta^{\prime}$ ) We can finally define $\delta^{\prime}$ to be the labelling of $\Gamma$ by (rank $1 \kappa$-terms of) $\Omega_{A}^{\kappa} \mathbf{S}$ such that $\mathrm{g} \delta^{\prime}=\widehat{u_{\mathrm{g} \delta}}$ for any $\mathrm{g} \in \Gamma$.

Notice that, by (13) to (18), $\mathrm{g} \delta^{\prime}$ was obtained in such a way that conditions $\mathrm{c} 2$ ) and c3) of Proposition 6.1 are satisfied. Moreover, the remarks made after the definition of the reduction rule R), in the end Section 6.2, clearly imply that $\mathrm{g} \delta^{\prime} \psi=u_{\mathrm{g} \delta} \psi$. Therefore, by Lemma 3.3, we deduce that $\mathrm{g} \delta^{\prime} \psi=\mathrm{g} \delta \psi$ and, so, that $\delta^{\prime} \psi=\gamma$. To establish condition c1) of Proposition 6.1, it remains to show that $\delta^{\prime} p_{\mathbf{L S I}}$ is consistent. This will be done in the next section where we also show that condition $\mathrm{c} 4$ ) of the same result holds.

\subsection{Proof of the consistency of $\delta^{\prime} p_{\text {LSI }}$}

To show that $\delta^{\prime} p_{\mathbf{L S l}}$ is consistent, we have to prove that e $\alpha \delta^{\prime} p_{\mathbf{L S} \mathbf{l}} \cdot \mathrm{e} \delta^{\prime} p_{\mathbf{L S l}}=\mathrm{e} \omega \delta^{\prime} p_{\mathbf{L S} \mathbf{I}}$ for every edge e. Let e be an arbitrary edge. Then, we have to prove that

$$
\left(\mathrm{e} \alpha \delta^{\prime} \cdot \mathrm{e} \delta^{\prime}\right) p_{\mathbf{L S} \mathbf{l}}=\mathrm{e} \omega \delta^{\prime} p_{\mathbf{L S} \mathbf{l}}
$$

By Proposition 5.1, this equality holds if and only if $\left(\mathrm{e} \alpha \delta^{\prime} \cdot \mathrm{e} \delta^{\prime}\right) p_{\mathbf{L I}}=\mathrm{e} \omega \delta^{\prime} p_{\mathbf{L I}}$ and $\mathrm{e} \alpha \delta^{\prime} \cdot \mathrm{e} \delta^{\prime}$ and $\mathrm{e} \omega \delta^{\prime}$ have the same bi-infinite factors. From (20) and (19), we have

$$
\begin{aligned}
\mathrm{e} \alpha \delta^{\prime} \cdot \mathrm{e} \delta^{\prime} & =\widehat{u_{\mathrm{e} \alpha} \delta} \cdot \widehat{u_{\mathrm{e}} \delta} \\
& =\widehat{l_{\mathrm{e} \alpha}} \widehat{u_{\mathrm{e} \alpha, 0}} \widehat{c_{\mathrm{e} \alpha, 1}} \cdots \widehat{c_{\mathrm{e} \alpha, s_{\mathrm{e} \alpha}}} \widehat{u_{\mathrm{e} \alpha, s_{\mathrm{e}} \alpha}} \widehat{r_{\mathrm{e} \alpha}} \cdot \widehat{l_{\mathrm{e}}} \widehat{u_{\mathrm{e}, 0}} \widehat{c_{\mathrm{e}, 1}} \cdots \widehat{c_{\mathrm{e}, s_{\mathrm{e}}}} \widehat{u_{\mathrm{e}, s_{\mathrm{e}}}} \widehat{r_{\mathrm{e}}} \\
& =\widehat{l_{\mathrm{e} \alpha}} \widehat{u_{\mathrm{e} \alpha, 0}} \widehat{c_{\mathrm{e} \alpha, 1}} \cdots \widehat{c_{\mathrm{e} \alpha, s_{\mathrm{e} \alpha}}} \widehat{u_{\mathrm{e} \alpha, s_{\mathrm{e} \alpha}}} \widehat{c_{\mathrm{e}}} \widehat{u_{\mathrm{e}, 0}} \widehat{c_{\mathrm{e}, 1}} \cdots \widehat{c_{\mathrm{e}, s_{\mathrm{e}}}} \widehat{u_{\mathrm{e}, s_{\mathrm{e}}}} \widehat{r_{\mathrm{e}}}
\end{aligned}
$$


and

$$
\mathrm{e} \omega \delta^{\prime}=\widehat{u_{\mathrm{e} \omega \delta}}=\widehat{l_{\mathrm{e} \omega}} \widehat{u_{\mathrm{e} \omega, 0}} \widehat{c_{\mathrm{e} \omega, 1}} \cdots \widehat{c_{\mathrm{e} \omega, s_{\mathrm{e}} \omega}} \widehat{u_{\mathrm{e} \omega, s_{\mathrm{e}}}} \widehat{\mathrm{e}_{\mathrm{e} \omega}} .
$$

Since e $\alpha$ and e $\omega$ belong to the same connected component of $\Gamma$, i.e., e $\alpha \in$ e $\omega \theta$, (8) implies that $l_{\mathrm{e} \omega}=l_{\mathrm{e} \alpha}$, so that $\widehat{l_{\mathrm{e} \omega}}=\widehat{\mathrm{e}_{\mathrm{e} \alpha}}$. Moreover, by (16), $\widehat{r_{\mathrm{e}}}=\widehat{r_{\mathrm{e} \omega}}$. Therefore $\left(\mathrm{e} \alpha \delta^{\prime} \cdot \mathrm{e} \delta^{\prime}\right) p_{\mathbf{L I}}=\mathrm{e} \omega \delta^{\prime} p_{\mathbf{L I}}$.

Let us now show that $\mathrm{e} \alpha \delta^{\prime} \cdot \mathrm{e} \delta^{\prime}$ and $\mathrm{e} \omega \delta^{\prime}$ have the same bi-infinite factors. That is, let us show that e $\alpha \delta^{\prime} \cdot \mathrm{e} \delta^{\prime}$ and $\mathrm{e} \omega \delta^{\prime}$ have the same factors of the form $x^{\omega}$ and $x^{\omega} y z^{\omega}$, with $x, z \in A^{+}$and $y \in A^{*}$. Since each of $\mathrm{e} \alpha \delta^{\prime} \cdot \mathrm{e} \delta^{\prime}$ and $\mathrm{e} \omega \delta^{\prime}$ has at least two occurrences of factors of the form $x^{\omega}$, it clearly suffices to show that they have the same factors of the form $x^{\omega} y z^{\omega}$. Moreover, since each occurrence of a factor $x^{\omega}$ appears in an occurrence of a factor of the form $\bar{u}$, it suffices to show that $\mathrm{e} \alpha \delta^{\prime} \cdot \mathrm{e} \delta^{\prime}$ and $\mathrm{e} \omega \delta^{\prime}$ have the same factors of the form

$$
\bar{x} y \bar{z}, \quad \text { with } x, z \in A^{+} \text {and } y \in A^{*} .
$$

Notice first that, since $(\mathrm{e} \alpha \delta \cdot \mathrm{e} \delta) q_{\mathbf{L S I}}=\mathrm{e} \omega \delta q_{\mathbf{L S} \mathbf{l}}$ by consistency of $\delta q_{\mathbf{L S} \mathbf{I}}$ and since $\equiv_{n}$ is a congruence on $\bar{\Omega}_{A} \mathbf{S}$, we deduce from Lemma 3.3 that $u_{\mathrm{e} \alpha \delta} \cdot u_{\mathrm{e} \delta} \equiv_{n} u_{\mathrm{e} \omega \delta}$. Let us now notice the following observations.

Lemma 6.9 The following statements hold.

a) $\left\{c_{\mathrm{e} \alpha, 1}, \ldots, c_{\mathrm{e} \alpha, s_{\mathrm{e} \alpha}}, c_{\mathrm{e}}, c_{\mathrm{e}, 1}, \ldots, c_{\mathrm{e}, s_{\mathrm{e}}}\right\}=\left\{c_{\mathrm{e} \omega, 1}, \ldots, c_{\mathrm{e} \omega, s_{\mathrm{e}}}\right\}$;

b) $u_{\mathrm{e} \alpha \delta} \cdot u_{\mathrm{e} \delta}$ and $u_{\mathrm{e} w \delta}$ have the same factors of length $3 k-1$ with relatively-free occurrences (we say that an occurrence of length $3 k-1$ is relatively-free in $u_{\mathrm{g} \delta}$ if it occurs in some $u_{\mathrm{g}, i}$, with $0 \leq i \leq s_{\mathrm{g}}$, and it is free in $\left.u_{\mathrm{g}, i}\right)$;

c) $u_{\mathrm{e} \alpha \delta} \cdot u_{\mathrm{e} \delta}$ and $u_{\mathrm{e} w \delta}$ have the same relatively-marked factors (we say that a factor of $u_{\mathrm{g} \delta}$ is relatively-marked if it is a marked factor in some $u_{\mathrm{g}, i}$ with $0 \leq i \leq s_{\mathrm{g}}$ ).

Proof. Let $i \in\left\{1, \ldots, s_{\mathrm{e} \omega}\right\}$. Then $c_{\mathrm{e} \omega, i}=c_{\mathrm{f}}$ for some edge $\mathrm{f}$, and $c_{\mathrm{f}}$ is a factor of $u_{\mathrm{e} \omega \delta}$. So, since $n \geq\left|c_{f}\right|$, we deduce from Lemma 3.3 and condition $(d)$ on Definition 6.4, that $\mathbf{w}_{\mathrm{f}}$ is a bi-infinite factor of e $\omega \delta$. Hence, by consistency of $\delta q_{\mathbf{L S l}}$, we deduce that $\mathbf{w}_{\mathrm{f}}$ is a bi-infinite factor of $\mathrm{e} \alpha \delta \cdot \mathrm{e} \delta$. Now, Proposition 3.2 implies that $\mathbf{w}_{\mathrm{f}}$ is a factor of $\mathrm{e} \alpha \delta$, or a factor of $\mathrm{e} \delta$, or $\mathbf{w}_{\mathrm{f}}$ is a shift of the bi-infinite word $\mathbf{w}_{\mathrm{e}}=\mathrm{e} \alpha \delta q_{\mathbf{D}} \cdot \mathrm{e} \delta q_{\mathbf{K}}$. Therefore, we conclude respectively that $c_{\mathrm{f}}$ is an allowed center of $u_{\mathrm{e} \alpha \delta}$ (if $c_{\mathrm{f}}$ is non-periodic, then $\overleftarrow{c_{\mathrm{f}}}$ is a factor of e $\alpha \delta$ and $\left|\overleftarrow{c_{\mathrm{f}}}\right| \leq L<n$ whence $\overleftarrow{c_{\mathrm{f}}}$ is also a factor of $\left.u_{\mathrm{e} \alpha \delta}\right)$, or that $c_{\mathrm{f}}$ is an allowed center of $u_{\mathrm{e} \delta}$ (analogously), or, by condition (a) on Definition 6.4, that $c_{\mathrm{f}}=c_{\mathrm{e}}$. This proves that $c_{\mathrm{e} \omega, i} \in\left\{c_{\mathrm{e} \alpha, 1}, \ldots, c_{\mathrm{e} \alpha, s_{\mathrm{e} \alpha}}, c_{\mathrm{e}}, c_{\mathrm{e}, 1}, \ldots, c_{\mathrm{e}, s_{\mathrm{e}}}\right\}$. Inversely, one deduces analogously that all centers $c_{\mathrm{e} \alpha, i}, c_{\mathrm{e}}$ and $c_{\mathrm{e}, j}$ are allowed centers of $u_{\mathrm{e} \omega \delta}$, since the corresponding bi-infinite words $\mathbf{w}_{\mathrm{f}}$ can be shown to be factors of e $\omega \delta$. This concludes the proof of statement $a$ ).

Let now $x$ be a factor of length $3 k-1$ with a relatively-free occurrence in $u_{\mathrm{e} \omega \delta}$. Then $x$ has a free occurrence $o$ in $u_{\mathrm{e} \omega, i}$, for some $i \in\left\{1, \ldots, s_{\mathrm{e} \omega}\right\}$. Therefore, by definition of free occurrence, there exists a $k^{\prime \prime}$-neighborhood $v$ of $o$ in $u_{\mathrm{e} \omega, i}$ such that $v$ is $k^{\prime}$-abundant. In particular, $|v| \leq k^{\prime \prime}$. Suppose that $y v z$ is an extension of $v$ in $u_{\mathrm{e}} \omega \delta$ with $|y|,|z|=L$. 
Then, $|y v z| \leq 2 L+k^{\prime \prime}<n$. Therefore $y v z$ has an occurrence in $u_{\mathrm{e} \alpha \delta} \cdot u_{\mathrm{e} \delta}$, since $u_{\mathrm{e} \omega \delta}$ and $u_{\mathrm{e} \alpha \delta} \cdot u_{\mathrm{e} \delta}$ have the same factors of length $n$. Then the corresponding occurrence of $v$ is disjoint from the allowed centers of $u_{\mathrm{e} \alpha \delta} \cdot u_{\mathrm{e} \delta}$. Indeed, if this was not the case, since the periodic centers and the extended-centers of non-periodic centers have length at most $L$, then the occurrence of $v$ in $u_{\mathrm{e} \omega \delta}$ would not be disjoint from the allowed centers of $u_{\mathrm{e} \omega \delta}$. So, in this case, $x$ has a free occurrence in $u_{\mathrm{e} \alpha \delta} \cdot u_{\mathrm{e} \delta}$. Suppose now that there is not an extension $y v z$ of $v$ in $u_{\mathrm{e} \omega \delta}$ with $|y|,|z|=L$. That is, if $y v z$ is an extension of $v$ in $u_{\mathrm{e} \omega \delta}$, then $|y|<L$ or $|z|<L$. In the first case, $v$ occurs in the prefix of $u_{\mathrm{e} w \delta}$ of length $L+k^{\prime \prime}$. Now, as $u_{\mathrm{e} w \delta}$ and $u_{\mathrm{e} \alpha \delta}$ have the same prefix of length $n>2 L+k^{\prime \prime}$, one can easily verify that the factorizations of $u_{\mathrm{e} \omega \delta}$ and $u_{\mathrm{e} \alpha \delta}$ for the centers of $\Gamma$ coincide on their comum prefix of length $L+k^{\prime \prime}$. Whence $v$ occurs in $u_{\mathrm{e} \alpha \delta}$ disjoint from $l_{\mathrm{e} \alpha}$ and from the centers $c_{\mathrm{e} \alpha, i}$, so that $x$ has a free occurrence in $u_{\mathrm{e} \alpha \delta}$ and so also in $u_{\mathrm{e} \alpha \delta} \cdot u_{\mathrm{e} \delta}$. The second case is symmetric. This concludes the proof that every factor of length $3 k-1$ that has a relatively-free occurrence in $u_{\mathrm{e} \omega \delta}$ has also a relatively-free occurrence in $u_{\mathrm{e} \alpha \delta} \cdot u_{\mathrm{e} \delta}$. The reverse could be proved symmetrically, whence statement $b$ ) is established.

The proof of $c$ ) can be made analogously. We just notice that $u_{\mathrm{e} \omega \delta}$ and $u_{\mathrm{e} \alpha \delta} \cdot u_{\mathrm{e} \delta}$ have the same factors of length $n$ and that the marked factors are of length $<k^{\prime \prime}$ by Lemma 4.6.

We are now able to show that $\mathrm{e} \alpha \delta^{\prime} \cdot \mathrm{e} \delta^{\prime}$ and $\mathrm{e} \omega \delta^{\prime}$ have the same factors of the form $\bar{x} y \bar{z}$. Let $\bar{x} y \bar{z}$ be an occurrence in $\mathrm{e} \alpha \delta^{\prime} \cdot \mathrm{e} \delta^{\prime}$. The following cases may arise:

- $\bar{x} y \bar{z}=\widehat{c}$ for some center $c \in\left\{c_{\mathrm{e} \alpha, 1}, \ldots, c_{\mathrm{e} \alpha, s_{\mathrm{e} \alpha}}, c_{\mathrm{e}}, c_{\mathrm{e}, 1}, \ldots, c_{\mathrm{e}, s_{\mathrm{e}}}\right\}$. By Lemma 6.9, $c \in\left\{c_{\mathrm{e} \omega, 1}, \ldots, c_{\mathrm{e} \omega, s_{\mathrm{e} \omega}}\right\}$. Therefore, $\bar{x} y \bar{z}$ is also a factor of e $\omega \delta^{\prime}$.

- $\bar{x} y \bar{z}$ is an occurrence in some $\widehat{u_{\mathrm{g}, i}}$ with $\mathrm{g} \in\{\mathrm{e} \alpha, \mathrm{e}\}$. Then, by definition of $\widehat{u_{\mathrm{g}, i}}$ in Section 6.5, either the occurrence $x y z \in A^{+}$is a marked factor in $u_{\mathrm{g}, i}$, or it is not contained in a marked factor (since, if $x y z$ is not a marked factor, then $\bar{x} y \bar{z}$ was obtained in the third or the fourth steps of the transformations of the word $u_{\mathrm{g}, i}$ ). If $x y z$ is a marked factor $w$ in $u_{\mathrm{g}, i}$, then by Lemma $6.9 x y z$ is a marked factor in some $u_{\mathrm{e} \omega, j}$. Therefore, by the first step in section $6.5, \bar{x} y \bar{z}=\widehat{w}$ is a factor of $\widehat{u_{\mathrm{e}} \omega, j}$ and so also of e $\omega \delta^{\prime}$.

Suppose now that the occurrence $x y z$ in $u_{\mathrm{g}, i}$ is not contained in a marked factor. Then $|y|<k$, so that $2 k \leq|x y z|<3 k$, and, by the third step in Section 6.5, there exists a free occurrence of $x y z$ in $u_{\mathrm{g}, i}$. Hence, by Lemma 6.9, $x y z$ has a free occurrence in some $u_{\mathrm{e} \omega, j}$ and so, by the third step, $\bar{x} y \bar{z}$ is a factor of $\widehat{u_{\mathrm{e} \omega, j}}$ and so also of e $\omega \delta^{\prime}$.

- $\bar{x} y \bar{z}$ is an occurrence not disjoint both with $\widehat{l_{\mathrm{e} \alpha}}$ and $\widehat{u_{\mathrm{e} \alpha}, 0}$. In this case, $\widehat{l_{\mathrm{e} \alpha}}=p \bar{x}$ for some $p \in A^{*}$ by (17) and (18). Hence, since $l_{\mathrm{e} \alpha}=l_{\mathrm{e} \omega}$, we also have $\widehat{l_{\mathrm{e} \omega}}=p \bar{x}$. On the other hand, let $u_{\mathrm{e} \alpha, 0}=x_{0} w_{1} x_{1} w_{2} \cdots w_{r} x_{r}$ and $u_{\mathrm{e} \omega, 0}=x_{0}^{\prime} w_{1}^{\prime} x_{1}^{\prime} w_{2}^{\prime} \cdots w_{s}^{\prime} x_{s}^{\prime}$ be, respectively, the marked factorizations of $u_{\mathrm{e} \alpha, 0}$ and $u_{\mathrm{e} \omega, 0}$.

If $\left|u_{\mathrm{e} \alpha, 0}\right|<3 k-1$, then $u_{\mathrm{e} \alpha, 0}=u_{\mathrm{e} \omega, 0}$ and $c_{\mathrm{e} \alpha, 1}=c_{\mathrm{e} \omega, 1}$ since $u_{\mathrm{e} \alpha \delta}$ and $u_{\mathrm{e} \omega \delta}$ have the same prefix of length $n>3 L$. Therefore, by the first step of the transformation 
described in Section 6.5,

$$
\widehat{u_{\mathrm{e} \alpha, 0}}=w_{1}=u_{\mathrm{e} \alpha, 0}=u_{\mathrm{e} \omega, 0}=w_{1}^{\prime}=\widehat{u_{\mathrm{e} \omega, 0}} .
$$

so that, $y=w_{1}=w_{1}^{\prime}$ and $\widehat{\mathrm{e} \alpha_{\mathrm{e}, 1}}=\widehat{\mathrm{e}_{\mathrm{e}, 1}}=\bar{z} s$ for some $s \in \bar{A}^{+}$. Therefore, $\bar{x} y \bar{z}$ is a factor of e $\omega \delta^{\prime}$.

Suppose now that $\left|u_{\mathrm{e} \alpha, 0}\right| \geq 3 k-1$. Then, by the second step of the transformation described in Section 6.5,yz is either $x_{0} \overline{w_{1,1}}$ if $\left|x_{0}\right|<k$, or $\overline{x_{0,1}}$ if $\left|x_{0}\right| \geq k$. Since $u_{\mathrm{e} \alpha \delta}$ and $u_{\mathrm{e} \omega \delta}$ have the same prefix of length $n$, if $\left|x_{0}\right|<k$, then $x_{0}^{\prime}=x_{0}$ and $w_{1}=w_{1}^{\prime}$. In this case $y \bar{z}=x_{0}^{\prime} \overline{w_{1,1}^{\prime}}=x_{0} \overline{w_{1,1}}$. If $\left|x_{0}\right| \geq k$, then $x_{0,1}^{\prime}=x_{0,1}$ and so $y \bar{z}=\overline{x_{0,1}^{\prime}}=\overline{x_{0,1}}$. Therefore, we deduce in both cases that $\bar{x} y \bar{z}$ is a factor of $\widehat{l_{\mathrm{e} \omega}} \widehat{u_{\mathrm{e} \omega, 0}}$, whence it is a factor of e $\omega \delta^{\prime}$.

- $\bar{x} y \bar{z}$ is an occurrence not disjoint both with $\widehat{u_{\mathrm{e}, s_{\mathrm{e}}}}$ and $\widehat{r_{\mathrm{e}}}$. This case is symmetric of the previous one.

- for some $1 \leq i \leq s_{\mathrm{g}}, \bar{x} y \bar{z}$ is an occurrence not disjoint both with $\widehat{u_{\mathrm{g}, i-1}}$ and $\widehat{c_{\mathrm{g}, i}}$, or with $\widehat{c_{\mathrm{g}, i}}$ and $\widehat{u_{\mathrm{g}, i}}$ (where $\mathrm{g} \in\{\mathrm{e} \alpha, \mathrm{e}\}$ ), or with $\widehat{u_{\mathrm{e} \alpha, s_{\mathrm{e} \alpha}}}$ and $\widehat{c_{\mathrm{e}}}$, or with $\widehat{c_{\mathrm{e}}}$ and $\widehat{u_{\mathrm{e}, 0}}$. These cases are similar to the two last ones.

Therefore, we have proved in all cases that $\bar{x} y \bar{z}$ is a factor of e $\omega \delta^{\prime}$. Analogously, one can show that every factor $\bar{x} y \bar{z}$ of e $\omega \delta^{\prime}$ is also a factor of e $\alpha \delta^{\prime} \cdot \mathrm{e} \delta^{\prime}$. This concludes the proof of the consistency of $\delta^{\prime} p_{\mathbf{L S I}}$ and establishes condition c1) of Proposition 6.1.

The proof of condition $\mathrm{c} 4$ ) can be made analogously. In fact, if $\mathrm{v}_{1}$ and $\mathrm{v}_{2}$ are vertices such that $\mathrm{v}_{1} \delta q_{\mathbf{L S I}}=\mathrm{v}_{2} \delta q_{\mathbf{L S l}}$, then, in particular, the words $u_{\mathrm{v}_{1} \delta}$ and $u_{\mathrm{v}_{2} \delta}$ are $\equiv_{n^{-}}$ equivalent by Proposition 3.2 and Lemma 3.3. Therefore, we can assume that $r_{\mathrm{v}_{1}}=r_{\mathrm{v}_{2}}$ so that we can also assume that $\widehat{l_{\mathrm{v}_{1}}}=\widehat{l_{\mathrm{v}_{2}}}$ and $\widehat{\mathrm{v}_{1}}=\widehat{\mathrm{r}_{2}}$. Moreover, one can show that the words $u_{\mathrm{v}_{1} \delta}$ and $u_{\mathrm{v}_{2} \delta}$ have the same allowed centers, the same factors of length $3 k-1$ with relatively-free occurrences and the same relatively-marked factors. The proof that $\mathbf{v}_{1} \delta^{\prime} p_{\mathbf{L S I}}=\mathrm{v}_{2} \delta^{\prime} p_{\mathbf{L S I}}$ is now analogous to the one above. By Proposition 6.1 , this proves the following result.

Proposition 6.10 The pseudovariety LSl is $\kappa$-reducible.

Finally we deduce the main result of this work.

Theorem 6.11 The pseudovariety LSl is $\kappa$-tame.

\section{References}

[1] D. Albert, R. Baldinger and J. Rhodes, The identity problem for finite semigroups (the undecidability of), J. Symbolic Logic 57 (1992), 179-192.

[2] J. Almeida, Finite semigroups and universal algebra, World Scientific, Singapore, 1995. English translation.

[3] J. Almeida, Hyperdecidable pseudovarieties and the calculation of semidirect products, Int. J. Algebra and Computation 9 (1999), 241-261. 
[4] J. Almeida, Some key problems on finite semigroups, Semigroup Forum 64 (2002), 159-179.

[5] J. Almeida, Finite semigroups: An Introduction to a unified theory of pseudovarieties, in Semigroups, Algorithms, Automata and Languages, ed. G. Gomes, World Scientific, 2002, pp. 3-64.

[6] J. Almeida and M. Delgado, Tameness of the pseudovariety of abelian groups, Int. J. Algebra and Computation, to appear.

[7] J. Almeida and B. Steinberg, On the decidability of iterated semidirect products and applications to complexity, Proc. London Math. Soc. 80 (2000), 50-74.

[8] J. Almeida and P. Weil, Relatively free profinite monoids: an introduction and examples, in Semigroups, Formal Languages and Groups, ed. J. Fountain, Kluwer, 1995, pp. 73-117.

[9] J. Almeida and M. Zeitoun, Tameness of some locally trivial pseudovarieties, Communications in Algebra 31 (2003), 61-77.

[10] C. Ash, Inevitable graphs: a proof of the type II conjecture and some related decision procedures, Int. J. Algebra and Computation 1 (1991), 127-146.

[11] J. Brozozowski and I. Simon, Characterization of locally testable events, Discrete Math. 4 (1973), 243-271.

[12] J. C. Costa, Free profinite locally idempotent and locally commutative semigroups, Journal of Pure and Applied Algebra 163 (2001), 19-47.

[13] J. C. Costa, Reducibility of joins involving some locally trivial pseudovarieties, Communications in Algebra, to appear.

[14] S. Eilenberg, Automata, languages and machines, vol.B, Academic Press, New York, 1976.

[15] K. Krohn and J. Rhodes, Algebraic theory of machines I - Prime decomposition theorem for finite semigroups and machines, Trans. Amer. Math. Soc. 116 (1965), 450-464.

[16] R. McNaughton, Algebraic decision procedures for local testability, Math. Systems Theory 8 (1974), 60-76.

[17] J.-E. Pin, Variétés de langages formels, Masson, Paris, 1984. English translation: Varieties of formal languages, Plenum, New York and North Oxford, London, 1986.

[18] J. Reiterman, The Birkhoff theorem for finite algebras, Algebra Universalis 14 (1982), 1-10.

[19] J. Rhodes, Undecidability, automata and pseudovarieties of semigroups, Int. J. Algebra and Computation 9 (1999), 455-473. 